\title{
Counting packings of generic subsets in finite groups
}

\author{
Roland Bacher \\ Université Grenoble I \\ CNRS UMR 5582, Institut Fourier \\ BP 74 \\ F-38402 St. Martin d'Hères, France \\ roland.bacher@ujf-grenoble.fr
}

Submitted: Sep 5, 2011; Accepted: Jul 6, 2012; Published: Jul 12, 2012

Mathematics Subject Classifications: 05A15, 05C30, 11B73, 11P99

\begin{abstract}
A packing of subsets $\mathcal{S}_{1}, \ldots, \mathcal{S}_{n}$ in a group $G$ is an element $\left(g_{1}, \ldots, g_{n}\right)$ of $G^{n}$ such that $g_{1} \mathcal{S}_{1}, \ldots, g_{n} \mathcal{S}_{n}$ are disjoint subsets of $G$. We give a formula for the number of packings if the group $G$ is finite and if the subsets $\mathcal{S}_{1}, \ldots, \mathcal{S}_{n}$ satisfy a genericity condition. This formula can be seen as a generalization of the falling factorials which encode the number of packings in the case where all the sets $\mathcal{S}_{i}$ are singletons.
\end{abstract}

Keywords: Enumerative combinatorics; packings in groups; additive combinatorics; additive number theory; Stirling number

\section{Introduction}

A (left-)packing of $n$ non-empty subsets $\mathcal{S}_{1}, \ldots, \mathcal{S}_{n}$ in a group $G$ is an element $\left(g_{1}, \ldots, g_{n}\right)$ of $G^{n}$ such that the left-translates $g_{1} \mathcal{S}_{1}, \ldots, g_{n} \mathcal{S}_{n}$ of the sets $\mathcal{S}_{i}$ are disjoint. The sets $\mathcal{S}_{1}, \ldots, \mathcal{S}_{n}$ are labelled by their indices. In particular, permuting the elements $g_{1}, \ldots, g_{n}$ of a packing $\left(g_{1}, \ldots, g_{n}\right) \in \mathcal{G}^{n}$ of $\mathcal{S}_{1}=\cdots=\mathcal{S}_{n}$ yields a different packing. Moreover, in the case where $\mathcal{S}_{1}$ for example is of the form $\mathcal{S}_{1}=H \mathcal{S}_{1}$ for some subgroup $H$ of $G$, a packing $\left(g_{1}, \ldots, g_{n}\right)$ gives rise to $\sharp(H)$ distinct packings $\left(g_{1} h, g_{2}, \ldots, g_{n}\right), h \in H$.

There is an obvious one-to-one map between packings of $\mathcal{S}_{1}, \ldots, \mathcal{S}_{n} \subset G$ and packings of $a_{1} \mathcal{S}_{1}, \ldots, a_{n} \mathcal{S}_{n} \subset G$ for every $\left(a_{1}, \ldots, a_{n}\right) \in G^{n}$.

This paper deals with enumerative properties of left-packings in the case where $G$ is a finite group. Using the involutive antiautomorphism $g \longmapsto g^{-1}$, its content can easily be modified in order to deal with right-packings $\mathcal{S}_{1} g_{1}, \ldots, \mathcal{S}_{n} g_{n}$.

In the sequel, we denote by $\alpha\left(G ; \mathcal{S}_{1}, \ldots, \mathcal{S}_{n}\right) \leqslant N^{n}$ the number of packings of $n$ nonempty subsets $\mathcal{S}_{1}, \ldots, \mathcal{S}_{n}$ in a finite group $G$ with $N$ elements. Computing $\alpha\left(G ; \mathcal{S}_{1}, \ldots, \mathcal{S}_{n}\right)$ 
for arbitrary subsets $\mathcal{S}_{1}, \ldots, \mathcal{S}_{n}$ in a finite group $G$ is probably difficult. There are however easy lower and upper bounds:

Proposition 1. We set $a=\alpha\left(G ; \mathcal{S}_{1}, \ldots, \mathcal{S}_{n}\right)$ and $b=\alpha\left(G ; \mathcal{S}_{1}, \ldots, \mathcal{S}_{n}, \mathcal{S}_{n+1}\right)$ where $\mathcal{S}_{1}, \ldots, \mathcal{S}_{n+1}$ are $(n+1)$ non-empty subsets in a finite group $G$. We have the inequalities

$$
\left(N-\sharp\left(\mathcal{S}_{n+1}\right) \sum_{i=1}^{n} \sharp\left(\mathcal{S}_{i}\right)\right) a \leqslant b \leqslant\left(N-\sum_{i=1}^{n} \sharp\left(\mathcal{S}_{i}\right)\right) a .
$$

In particular, we have

$$
b=\left(N-\sum_{i=1}^{n} \sharp\left(\mathcal{S}_{i}\right)\right) a
$$

if $\mathcal{S}_{n+1}$ is a singleton.

Proposition 1 will be proven in Section 3.

A family $\mathcal{S}_{1}, \ldots, \mathcal{S}_{n}$ of $n$ non-empty subsets in a group $G$ with identity element $e$ is generic if for every sequence $i_{1}, \ldots, i_{k}$ of $k$ distinct elements in $\{1, \ldots, n\}$ and for every choice of elements $g_{i_{j}} \in \mathcal{S}_{i_{j}}^{-1} \mathcal{S}_{i_{j}} \backslash\{e\}$, we have

$$
g_{i_{1}} g_{i_{2}} \cdots g_{i_{k}} \neq e .
$$

Otherwise stated, a family $\mathcal{S}_{1}, \ldots, \mathcal{S}_{n}$ of subsets in a group $G$ is generic if the only solution of the equations $g_{i_{1}} \cdots g_{i_{n}}=e$ with $g_{i_{j}} \in \mathcal{S}_{i_{j}}^{-1} \mathcal{S}_{i_{j}}$ for $\left\{i_{1}, \ldots, i_{n}\right\}=\{1, \ldots, n\}$ is given by $g_{i_{j}}=e$ for all $j$.

Genericity excludes "accidental intersections" among translates $g_{1} \mathcal{S}_{1}, \ldots, g_{n} \mathcal{S}_{n}$ in the following sense: Given a collection of translates $g_{1} \mathcal{S}_{1}, \ldots, g_{n} \mathcal{S}_{n}$, we consider the associated intersection graph with vertices $\mathcal{S}_{i}$ and edges joining $\mathcal{S}_{i}, \mathcal{S}_{j}$ if $g_{i} \mathcal{S}_{i} \cap g_{j} \mathcal{S}_{j} \neq \emptyset$. Genericity of a family $\mathcal{S}_{1}, \ldots, \mathcal{S}_{j}$ in a group $G$ is equivalent to the statement that all intersection graphs are primal graphs of hyperforests. Intuitively speaking, intersections among translates of a generic family are always "as small as possible".

Example 2. Genericity in an additive abelian group $G$ boils down to the fact that the subset $\left(\mathcal{S}_{1}-\mathcal{S}_{1}\right) \times \cdots \times\left(\mathcal{S}_{n}-\mathcal{S}_{n}\right)$ of the group $G^{n}$ intersects the subgroup $\left\{\left(x_{1}, \ldots, x_{n}\right) \in\right.$ $\left.G^{n} \mid \sum_{i=1}^{n} x_{i}=0\right\}$ of $G^{n}$ only in the identity element $(0, \ldots, 0)$.

A generic family $\mathcal{S}_{1}, \ldots, \mathcal{S}_{n}$ of subsets in the additive group $\mathbb{Z}$ with prescribed cardinalities $s_{i}=\sharp\left(\mathcal{S}_{i}\right)$ can be constructed by starting with $\mathcal{S}_{1}=\left\{0, \ldots, s_{1}-1\right\}$ and by defining $\mathcal{S}_{i}$ recursively as $\mathcal{S}_{i}=\left\{0, k_{i}, 2 k_{i}, \ldots,\left(s_{i}-1\right) k_{i}\right\}$ where $k_{i}$ is an arbitrary natural integer strictly larger than $\sum_{j=1}^{i-1}\left(\max \left(\mathcal{S}_{j}\right)-\min \left(\mathcal{S}_{j}\right)\right)=\sum_{j=1}^{i-1}\left(s_{j}-1\right) k_{j}$. A generic family is thus for example given by the sets $\mathcal{S}_{1}=\{0,1\}, \mathcal{S}_{2}=\{0,2\}, \ldots, \mathcal{S}_{i}=\left\{0,2^{i-1}\right\}, \ldots, \mathcal{S}_{n}=\left\{0,2^{n-1}\right\}$.

Reduction of a generic family $\mathcal{S}_{1}, \ldots, \mathcal{S}_{n} \subset \mathbb{Z}$ modulo a natural integer $N$ yields a generic family of $\mathbb{Z} / N \mathbb{Z}$ except if $N$ is a divisor of a non-zero integer in the finite set $\left\{\sum_{i=1}^{n}\left(\mathcal{S}_{i}-\mathcal{S}_{i}\right)\right\}$. 
Remark 3. The terminology "generic family" can be motivated as follows: Given $n$ strictly positive natural numbers $s_{1}, \ldots, s_{n}$, most uniform random choices of $n$ subsets $\mathcal{S}_{1}, \ldots, \mathcal{S}_{n}$ with $\sharp\left(\mathcal{S}_{i}\right)=s_{i}$ (among all $\left(\begin{array}{l}N \\ s_{i}\end{array}\right)$ possible subsets) in a finite group $G$ of order $N$ should yield a generic family if $N$ is large compared to $\sum_{k=2}^{n} k ! \tau_{k}$ with $\tau_{2}, \ldots, \tau_{n}$ defined by $\sum_{k=0}^{n} \tau_{k} t^{k}=\prod_{j=1}^{n}\left(1+s_{j}\left(s_{j}-1\right) t\right)$. Indeed, the number $k ! \tau_{k}$ is an upper bound on the number of elements in the set $E_{k}$ containing all products of the form $g_{i_{1}} \cdots g_{i_{k}}$ with $g_{i_{j}} \in \mathcal{S}_{i_{j}}^{-1} \mathcal{S}_{i_{j}} \backslash\{e\}$ and $i_{1}, \ldots, i_{k}$ given by $k \geqslant 2$ distinct elements of $\{1, \ldots, n\}$. Under the (naive but hopefully correct) assumption that the elements of $E_{k}$ are uniformly distributed in $G$, the probability for non-genericity of $\mathcal{S}_{1}, \ldots, \mathcal{S}_{n}$ is at most $\frac{1}{N} \sum_{k=2}^{n} k ! \kappa_{k}$. Observe also the trivial inequalities $\sum_{k=2}^{n} k ! \kappa_{k}<n ! \sum_{k=0}^{n} \kappa_{k}=n ! \prod_{j=1}^{n}\left(1+s_{j}\left(s_{j}-1\right)\right) \leqslant n ! \prod_{j=1}^{n} s_{j}^{2}$.

The aim of this paper is to describe a universal formula for the number of packings for a generic family of subsets $\mathcal{S}_{1}, \ldots, \mathcal{S}_{n}$ in a finite group $G$. The number of associated packings depends then only on the cardinalities of $G$ and $\mathcal{S}_{1}, \ldots, \mathcal{S}_{n}$. Moreover for fixed cardinalities of $\mathcal{S}_{1}, \ldots, \mathcal{S}_{n}$, the dependency on the cardinality of $G$ is polynomial of degree $n$. A trivial example is the generic family given by $n$ subsets reduced to singletons. The associated number of packings in a finite group with $N$ elements is then easily seen to be given by the polynomial $n !\left(\begin{array}{l}N \\ n\end{array}\right)=N(N-1) \cdots(N-n+1) \in \mathbb{Z}[N]$ with coefficients given by Stirling numbers of the first kind. This polynomial is also called a falling factorial and denoted by $N \underline{n}$. Using the formulae of our paper, it is possible to define the falling factorial $N^{\underline{\lambda}}$ associated to a partition $\lambda=\lambda_{1}, \lambda_{2}, \ldots$ by counting packings of generic families with $\lambda_{1}$ subsets having $\nu_{1}, \nu_{2}, \ldots, \nu_{\lambda_{1}}$ elements where $\nu_{i}=\left\{j \mid \lambda_{j} \geqslant i\right\}$ is the $i$-th part of the transposed partition $\nu=\lambda^{t}$ of $\lambda$. The map $\lambda \longmapsto N^{\underline{\lambda}}$ is however perhaps not exceedingly interesting. On one hand, it is not into since $N^{\underline{\lambda}}=N$ for every partition $\lambda$ of the form $1,1,1, \ldots$ On the other hand, fixing the content $\sum_{j} \lambda_{j}$ of the partition $\lambda$, our formulae show that the coefficients of $N \underline{\lambda}$ depend linearly on the elementary symmetric functions $\sigma_{2}=\sum_{i<j} \nu_{i} \nu_{j}, \sigma_{3}=\sum_{i<j<k} \nu_{i} \nu_{j} \nu_{k}, \ldots, \sigma_{\lambda_{1}}=\nu_{1} \nu_{2} \cdots \nu_{\lambda_{1}}$ of the partition $\nu=\lambda^{t}$.

The study of generic packings in groups is, as far as I am aware, a new addition to the already large set of classical notions of packings. Well-known and well-studied examples are lattice-packings in Euclidean spaces or more generally sphere-packings in metric spaces. Error-correcting codes corresponding to packings of spheres (with respect to the Hamming distance given by the number of distinct coordinates) into $\mathbb{F}_{q}^{d}$ are discrete analogues. The associated theories have however a different flavour since one tries to pack a huge (perhaps infinite) number of identical copies of spheres as tightly as possible.

Subsets in generic families are in general all distinct: Repetition destroys genericity except in the case of singletons. Moreover, packings of generic sets have typically very small densities. Generic families are mainly interesting for enumerative properties of the corresponding packings.

This paper is organized as follows: Section 2 contains the main result, Theorem 4 . It expresses the number of packings of a generic family $\mathcal{S}_{1}, \ldots, \mathcal{S}_{n}$ in a finite group in terms of a formal power series $U=U\left(x, \sigma_{1}, \sigma_{2}, \ldots\right) \in A[[x]]$ with coefficients in the ring $A=\mathbb{Z}\left[\sigma_{1}, \sigma_{2}, \ldots\right]$ of polynomials in elementary symmetric functions $\sigma_{1}=\sum_{i=1}^{n} \sharp\left(\mathcal{S}_{i}\right), \sigma_{2}=$ $\sum_{i<j} \sharp\left(\mathcal{S}_{i}\right) \sharp\left(\mathcal{S}_{j}\right), \ldots$ of $\sharp\left(\mathcal{S}_{1}\right), \ldots, \sharp\left(\mathcal{S}_{n}\right)$. The series $U$ is given explicitly by Formula 4 and involves combinatorial integers $t_{i, j}(n)$ (defined recursively by Formula 2) which extend 
Stirling numbers of the first kind. The first few coefficients of $U$ are given by

$$
\begin{aligned}
& 1-\sigma_{2} x-\left(\left(1-\sigma_{1}\right) \sigma_{3}+\sigma_{4}\right) x^{2} \\
& -\left(\left(2-3 \sigma_{1}+\sigma_{1}^{2}\right) \sigma_{4}+\left(5-3 \sigma_{1}\right) \sigma_{5}+3 \sigma_{6}\right) x^{3} \\
& -\left(\left(6-11 \sigma_{1}+6 \sigma_{1}^{2}-\sigma_{1}^{3}\right) \sigma_{5}+\left(26-26 \sigma_{1}+6 \sigma_{2}^{2}\right) \sigma_{6}\right. \\
& \left.\quad+\left(35-15 \sigma_{1}\right) \sigma_{7}+15 \sigma_{8}\right) x^{4}+\ldots
\end{aligned}
$$

with omitted terms divisible by $x^{5}$.

Section 3 discusses the combinatorics of packings associated to an arbitrary (not necessarily generic) family $\mathcal{S}_{1}, \ldots, \mathcal{S}_{n}$ of subsets in a group.

In Section 4, we specialize the results of Section 3 by applying them to generic packings. The underlying combinatorics are then simpler and yield a proof of Proposition 5, a crucial ingredient for establishing the main result.

Sections 5 and 6 contain the proof of Propositions 7 and 8 thus completing the proof of Theorem 4.

Section 7 uses Theorem 4 and its proof for computing the Möbius function of the poset of finite labelled hyperforests. An anonymous referee pointed out that this computation, a byproduct arising in the proof of our main result, might be of independent interest. Remark 22 states already known formulae for the enumeration of (weighted) labelled hypertrees.

Section 8 deals with computational aspects and examples.

Section 9 contains a conjectural asymptotic formula for the coefficients of the series $U(x, 0,-1,-1,-1, \ldots)$.

Section 10 describes a few experimental observations concerning arithmetical properties of the coefficients of $U(x, 0,-1,-1,-1, \ldots)$.

Section 11 is also experimental and describes a few integer sequences related to the numbers $t_{i, j}(n)$ appearing, up to signs, as coefficients of the series $U$.

The paper ends with Section 12 discussing a few aspects of coverings which can be seen as dual objects of packings.

\section{Main result}

For $n=1,2, \ldots$, we consider the following set $t_{i, j}(n)$ of strictly positive integers indexed by $i \in\{n+1, \ldots, 2 n\}$ and $j \in\{0,1, \ldots, 2 n-i\}$ : We set $t_{2,0}(1)=1$ and define $t_{i, j}(n)$ recursively by the formula

$$
t_{i, j}(n)=(i-2) t_{i-1, j}(n-1)+t_{i-1, j-1}(n-1)+(i-3) t_{i-2, j}(n-1)
$$

for $n \geqslant 2$. We set $t_{i, j}(n)=0$ in all other cases, i.e. if $i \leqslant n$ or $j<0$ or $i+j>2 n$.

Given a natural integer $n \geqslant 1$, the set of all $\left(\begin{array}{c}n+1 \\ 2\end{array}\right)$ non-zero integers $t_{i, j}(n)$ can be organized into a triangular array $T(n)$ with rows indexed by $\{n+1, \ldots, 2 n\}$ and columns indexed by $\{0, \ldots, n-1\}$ such that $T(n)$ determines $T(n+1)$ recursively by Formula 2 
reminiscent of the recurrence relation $\left(\begin{array}{l}n \\ k\end{array}\right)=\left(\begin{array}{c}n-1 \\ k-1\end{array}\right)+\left(\begin{array}{c}n-1 \\ k\end{array}\right)$ for binomial coefficients. The first six triangular arrays $T(1), \ldots, T(6)$ are

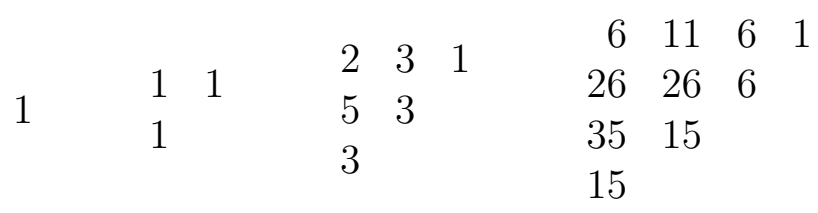

\begin{tabular}{|c|c|c|c|c|c|c|c|}
\hline 50 & & & 1 & 120 & 274 & 225 & 85 \\
\hline חסטר & & & & 1044 & 1604 & 855 & 190 \\
\hline $\begin{array}{l}200 \\
255\end{array}$ & 80 & 10 & & 3304 & 3325 & 1050 & 105 \\
\hline $\begin{array}{ll}40 & 255 \\
15 & 105\end{array}$ & 45 & & & 4900 & 2940 & 420 & \\
\hline 100 & & & & 3465 & 945 & & \\
\hline & & & & 945 & & & \\
\hline
\end{tabular}

Observe that the first row of $T(1), T(2), \ldots$ coincides, up to signs, with Stirling numbers of the first kind. More precisely, we have

$$
\sum_{k=0}^{n-1} t_{n+1, k}(n) x^{k+1}=\prod_{j=0}^{n-1}(x+j)=(-1)^{n} \sum_{j=1}^{n} S_{1}(n, j)(-x)^{j} .
$$

This is of course an easy consequence of the recurrence relation 2 . The integers $t_{i, j}(n)$ seem to be related to a few interesting integer-sequences, see Section 11 for examples.

We consider the formal power series $U \in A[[x]]$ with coefficients in the ring $A=$ $\mathbb{Z}\left[\sigma_{1}, \sigma_{2}, \sigma_{3}, \ldots\right]$ of integral polynomials in $\sigma_{1}, \sigma_{2}, \ldots$ defined by

$$
U\left(x, \sigma_{1}, \sigma_{2}, \ldots\right)=1-\sum_{n=1}^{\infty} x^{n} \sum_{i=n+1}^{2 n} \sigma_{i} \sum_{j=0}^{2 n-i} t_{i, j}(n)\left(-\sigma_{1}\right)^{j} .
$$

Theorem 4. The number of packings of a generic family $\mathcal{S}_{1}, \ldots, \mathcal{S}_{n}$ of $n$ non-empty subsets in a finite group $G$ with $N$ elements equals

$$
N^{n} U\left(N^{-1}, \sigma_{1}, \sigma_{2}, \ldots\right)
$$

for $U$ given by Formula 4 and for $\sigma_{1}, \sigma_{2}, \ldots$ defined by

$$
\sum_{j=0}^{\infty} \sigma_{j} t^{j}=\prod_{k=1}^{n}\left(1+\sharp\left(\mathcal{S}_{k}\right) t\right) .
$$

Remark that Formula 5 of Theorem 4 is polynomial of degree $n$ in $N$ for fixed complex numbers $\sigma_{1}, \sigma_{2}, \ldots$ such that $\sigma_{n+1}=\sigma_{n+2}=\cdots=0$. Indeed, the coefficient of $x^{m}$ in $U\left(x, \sigma_{1}, \sigma_{2}, \ldots\right)$ belongs to the ideal generated by $\sigma_{m+1}, \sigma_{m+2}, \ldots, \sigma_{2 m}$ of $\mathbb{Z}\left[\sigma_{1}, \sigma_{2}, \ldots\right]$ and is thus zero for $m \geqslant n$ if $\sigma_{n+1}=\sigma_{n+2}=\cdots=0$.

The ingredients for proving Theorem 4 are the following four results: 
Proposition 5. There exists a series $U \in \mathbb{Z}\left[\left[x, \sigma_{1}, \sigma_{2}, \ldots\right]\right]$ such that Formula 5 with $\sigma_{1}, \sigma_{2}, \ldots$ defined as in Theorem 4 gives the number of packings for every generic family of $n$ non-empty subsets in a finite group with $N$ elements.

Moreover, the coefficient of a non-constant monomial $x^{m}$ in this series $U$ is of degree at most $2 m$ with respect to the grading $\operatorname{deg} \sigma_{i}=i$ and belongs to the ideal of $\mathbb{Z}\left[\sigma_{1}, \sigma_{2}, \ldots\right]$ generated by $\sigma_{m+1}, \sigma_{m+2}, \ldots, \sigma_{2 m}$.

The proof of Proposition 5 relies on combinatorial properties of intersection graphs encoding non-trivial intersections among subsets $g_{1} \mathcal{S}_{1}, \ldots, g_{n} \mathcal{S}_{n}$ of a group $G$. These properties are encoded by the poset $\mathcal{H} \mathcal{F}(n)$ of hyperforests with $n$ labelled vertices and order relation given by $F^{\prime} \leqslant F$ if every hyperedge of $F^{\prime}$ is contained in some hyperedge of $F$. The poset $\mathcal{H} \mathcal{F}(n)$ is a lattice with minimal element the trivial graph defined by $n$ isolated labelled vertices and with maximal element the hypertree consisting of a unique hyperedge containing all $n$ labelled vertices. Our proof of Proposition 5 uses Möbius inversion in $\mathcal{H} F(n)$. It needs only the existence (which is obvious) of a Möbius function on the poset $\mathcal{H} \mathcal{F}(n)$. The explicit description of $U$ given by Theorem 4 allows however a posteriori the computation (given by Proposition 19) of the Möbius function of $\mathcal{H} \mathcal{F}(n)$. Remark that the poset $\mathcal{H} \mathcal{T}_{n}$ of hypertrees with $n$ labelled vertices appearing for example in [3] is a subposet of the order dual of $\mathcal{H} \mathcal{F}(n)$ obtained by restricting the inverse order of $\mathcal{H} \mathcal{F}(n)$ to the subset of all hypertrees in $\mathcal{H} \mathcal{F}(n)$.

Proposition 6. A series $U$ as in Proposition 5 satisfies the functional equation

$$
\left(1-\sigma_{1} x\right) U\left(x, \sigma_{1}, \sigma_{2}, \sigma_{3}, \ldots\right)=U\left(x, \tilde{\sigma}_{1}, \tilde{\sigma}_{2}, \tilde{\sigma}_{3}, \ldots\right)
$$

where $\tilde{\sigma}_{i}=\sigma_{i-1}+\sigma_{i}$, using the convention $\sigma_{0}=1$.

Proof. Equation 6 corresponds to equation 1 if $\sigma_{1}, \sigma_{2}, \ldots$ are elementary symmetric functions of a finite set of natural integers. The general case follows by remarking that the algebra of symmetric polynomials is a free polynomial algebra on the set of elementary symmetric polynomials.

Proposition 7. The series $U$ defined by Formula 4 satisfies the functional equation 6.

Proposition 8. The functional equation 6 has at most one solution of the form $U=$ $1+\ldots$ such that the coefficient of a nonconstant monomial $x^{n}$ is of degree at most $2 n$ (with respect to the grading $\operatorname{deg} \sigma_{i}=i$ ) and belongs to the ideal generated by $\sigma_{n+1}, \sigma_{n+2}, \ldots, \sigma_{2 n}$ in $\mathbb{Z}\left[\sigma_{1}, \sigma_{2}, \ldots\right]$.

Proof of Theorem 4. Proposition 5 ensures the existence of a series enumerating packings of generic families in finite groups. This series coincides with the series given by Formula 4 by Propositions 6, 7 and 8 .

Remark 9. Iterating identity $6 n$ times we have

$$
U\left(x, \sigma_{1}, \sigma_{2}, \ldots\right) \prod_{j=0}^{n-1}\left(1-\left(\sigma_{1}+j\right) x\right)=U\left(x, \tilde{\sigma}_{1}, \tilde{\sigma}_{2}, \tilde{\sigma}_{3}, \ldots\right)
$$


where

$$
\tilde{\sigma}_{k}=\sum_{j=0}^{\min (k, n)}\left(\begin{array}{l}
n \\
j
\end{array}\right) \sigma_{k-j} .
$$

A particular case is the specialization

$$
U\left(x,\left(\begin{array}{l}
n \\
1
\end{array}\right),\left(\begin{array}{l}
n \\
2
\end{array}\right),\left(\begin{array}{l}
n \\
3
\end{array}\right), \ldots\right)=\prod_{j=1}^{n-1}(1-j x)
$$

associated to generic families $\mathcal{S}_{1}, \ldots, \mathcal{S}_{n}$ given by $n$ singletons.

Remark 10. It is widespread lore that interesting combinatorial identities have $q$-analogues generally encoding an additional feature of the involved combinatorial objects. I do not know if the integers $t_{i, j}(n)$ or the series $U$ have such a $q$-analogue with interesting properties.

\section{Combinatorics of packings for arbitrary families $\mathcal{S}_{1}, \ldots, \mathcal{S}_{n}$ of subsets in a group $G$}

\subsection{Proof of Proposition 1}

Proof of Proposition 1. A packing of $\mathcal{S}_{1}, \ldots, \mathcal{S}_{n}$ given by $\left(g_{1}, \ldots, g_{n}\right) \in G^{n}$ extends to a packing $\left(g_{1}, \ldots, g_{n}, g_{n+1}\right) \in G^{n+1}$ of $\mathcal{S}_{1}, \ldots, \mathcal{S}_{n+1}$ if and only if $g_{n+1} \in G \backslash\left(\cup_{i=1}^{n} g_{i} \mathcal{S}_{i}\left(\mathcal{S}_{n+1}\right)^{-1}\right)$ where $\mathcal{S}^{-1}=\left\{g^{-1} \mid g \in \mathcal{S}\right\}$. Since $g_{i} \mathcal{S}_{i}\left(\mathcal{S}_{n+1}\right)^{-1}$ contains at most $\sharp\left(\mathcal{S}_{n+1}\right) \sharp\left(\mathcal{S}_{i}\right)$ elements, we have the first inequality.

Considering a fixed element $h \in \mathcal{S}_{n+1}$ we have the inequality

$$
\sharp\left(\cup_{i=1}^{n} g_{i} \mathcal{S}_{i}\left(\mathcal{S}_{n+1}\right)^{-1}\right) \geqslant \sharp\left(\cup_{i=1}^{n} g_{i} \mathcal{S}_{i} h^{-1}\right)=\sharp\left(\cup_{i=1}^{n} g_{i} \mathcal{S}_{i}\right) .
$$

For a packing $\left(g_{1}, \ldots, g_{n}\right)$, we have

$$
\sharp\left(\cup_{i=1}^{n} g_{i} \mathcal{S}_{i}\right)=\sum_{i=1}^{n} \sharp\left(\mathcal{S}_{i}\right)
$$

showing the second inequality.

Both inequalities are sharp if $\sharp\left(\mathcal{S}_{n+1}\right)=1$. This proves equality 1 .

\subsection{Intersection graphs}

We fix a group $G$ and a family $\mathcal{S}_{1}, \ldots, \mathcal{S}_{n}$ of $n$ non-empty subsets in $G$. Given an element $\mathbf{g}=\left(g_{1}, \ldots, g_{n}\right)$ of $G^{n}$, we consider the corresponding intersection graph $\mathcal{I}(\mathbf{g})$ with vertices $1, \ldots, n$ and edges $\{i, j\}$ between distinct vertices $i, j$ if $g_{i} \mathcal{S}_{i} \cap g_{j} \mathcal{S}_{j} \neq \emptyset$ in $G$. Observe that $\mathbf{g}=\left(g_{1}, \ldots, g_{n}\right)$ in $G^{n}$ defines a packing if and only if $\mathcal{I}(\mathbf{g})$ is the trivial graph with $n$ isolated vertices. 
Given a finite simple graph $\Gamma$ with vertices $1, \ldots, n$ and edges $E(\Gamma)$, we consider the set

$$
\mathcal{R}_{\Gamma}=\left\{\left(g_{1}, \ldots, g_{n}\right) \in G^{n} \mid g_{i} \mathcal{S}_{i} \cap g_{j} \mathcal{S}_{j} \neq \emptyset \text { for every }\{i, j\} \in E(\Gamma)\right\} .
$$

An element $\mathbf{g}$ in $G^{n}$ belongs thus to $\mathcal{R}_{\Gamma}$ if and only if $\Gamma$ is a subgraph of the intersection graph $\mathcal{I}(\mathbf{g})$.

We denote by $\mathcal{E}_{\Gamma}$ the set of equivalence classes of $\mathcal{R}_{\Gamma}$ defined by $\left(g_{1}, \ldots, g_{n}\right) \sim$ $\left(h_{1}, \ldots, h_{n}\right)$ if $g_{i} h_{i}^{-1}=g_{j} h_{j}^{-1}$ for every edge $\{i, j\}$ of $\Gamma$. Two elements $\mathbf{g}=\left(g_{1}, \ldots, g_{n}\right)$ and $\mathbf{h}=\left(h_{1}, \ldots, h_{n}\right)$ of $\mathcal{R}_{\Gamma}$ represent thus the same equivalence class of $\mathcal{E}_{\Gamma}$ if and only if the map $i \longmapsto g_{i} h_{i}^{-1}$ is constant on (vertices of) connected components.

Proposition 11. Suppose that $G$ is a finite group with $N$ elements. We have then

$$
\sharp\left(\mathcal{R}_{\Gamma}\right)=\sharp\left(\mathcal{E}_{\Gamma}\right) N^{c(\Gamma)}
$$

where $c(\Gamma)$ denotes the number of connected components of $\Gamma$.

Proof. We set $c=c(\Gamma)$ and we denote the connected components of $\Gamma$ by $\Gamma_{1}, \ldots, \Gamma_{c}$. We get a free action of $G^{c}$ on $\mathcal{R}_{\Gamma}$ by considering

$$
\left(a_{1}, \ldots, a_{c}\right) \cdot\left(g_{1}, \ldots, g_{n}\right) \longmapsto\left(a_{\gamma(1)}^{-1} g_{1}, \ldots, a_{\gamma(n)}^{-1} g_{n}\right)
$$

where $\gamma(i) \in\{1, \ldots, c\}$ is defined by the inclusion of the vertex $i$ in the $\gamma(i)$-th connected component $\Gamma_{\gamma(i)}$ of $\Gamma$. Orbits in $\mathcal{R}_{\Gamma}$ of this action are thus in one-to-one correspondence with equivalence classes of $\mathcal{E}_{\Gamma}$.

Remark 12. The set $\mathcal{E}_{\Gamma}$ associated to a graph $\Gamma$ with $c$ connected components contains at most $\left(\max _{i} \sharp\left(\mathcal{S}_{i}\right)\right)^{2 n-2 c}$ distinct equivalence classes. Indeed, we have $\mathcal{R}\left(\Gamma^{\prime}\right) \subset \mathcal{R}(\Gamma)$ if $\Gamma$ is a subgraph of $\Gamma^{\prime}$. Replacing $\Gamma$ by a spanning forest, we can thus assume that $\Gamma$ is a forest. The equivalence class of an element $\mathbf{g} \in \mathcal{R}(\Gamma)$ is now determined by the relative positions of $g_{i} \mathcal{S}_{i}$ and $g_{j} \mathcal{S}_{j}$ for all $n-c$ edges $\{i, j\}$ of the forest $\Gamma$ and the number of different relative positions of $g_{i} \mathcal{S}_{i}$ and $g_{j} \mathcal{S}_{j}$ is at most $\sharp\left(\mathcal{S}_{i}\right) \sharp\left(\mathcal{S}_{j}\right) \leqslant\left(\max _{i} \sharp\left(\mathcal{S}_{i}\right)\right)^{2}$.

\subsection{Möbius inversion}

Proposition 13. The number $\alpha=\alpha\left(G ; \mathcal{S}_{1}, \ldots, \mathcal{S}_{n}\right)$ of packings of a family $\mathcal{S}_{1}, \ldots, \mathcal{S}_{n}$ in a finite group $G$ with $N$ elements is given by

$$
\alpha=\sum_{\Gamma \in \mathcal{B}}(-1)^{e(\Gamma)} \sharp\left(\mathcal{E}_{\Gamma}\right) N^{c(\Gamma)}
$$

where the sum is over the Boolean poset $\mathcal{B}$ of all $2^{\left(\begin{array}{c}n \\ 2\end{array}\right)}$ simple graphs with vertices $1, \ldots, n$ and where $e(\Gamma)=\sharp(E(\Gamma))$, respectively $c(\Gamma)$, denotes the number of edges, respectively connected components, of a graph $\Gamma \in \mathcal{B}$. 
Proof. Proposition 11 shows that it is enough to prove the equality

$$
\alpha=\sum_{\Gamma \in \mathcal{B}}(-1)^{e(\Gamma)} \sharp\left(\mathcal{R}_{\Gamma}\right) .
$$

An element $\mathbf{g}=\left(g_{1}, \ldots, g_{n}\right) \in G^{n}$ defines a packing if and only if its intersection graph $\mathcal{I}(\mathbf{g})$ is trivial. It provides thus a contribution of 1 to $\alpha$ in this case since it is only involved as an element of $\mathcal{R}_{\Gamma}$ if $\Gamma$ is the trivial graph with isolated vertices $1, \ldots, n$ and no edges.

An element $\mathbf{g}=\left(g_{1}, \ldots, g_{n}\right) \in G^{n}$ with non-trivial intersection graph $\mathcal{I}(\mathbf{g})$ containing $e \geqslant 1$ edges yields a contribution of 0 to $\alpha$ since contributions coming from the $2^{e-1}$ subgraphs of $\mathcal{I}(\mathrm{g})$ containing an even number of edges cancel out with contributions associated to the $2^{e-1}$ subgraphs having an odd number of edges.

Remark 14. Introducing

$$
\alpha_{\Gamma}=\left\{\mathbf{g} \in G^{n} \mid \mathcal{I}(\mathbf{g})=\Gamma\right\},
$$

we have $\alpha=\alpha_{T}$ where $T$ denotes the trivial graph with $n$ isolated vertices $1, \ldots, n$. Our proof of Proposition 13 computes $\alpha$ by applying Möbius inversion (more precisely, its dual form, see Proposition 3.7.2 of [5])

$$
\alpha=\sum_{\Gamma \in \mathcal{B}} \mu(\Gamma) \sharp\left(\mathcal{R}_{\Gamma}\right)
$$

(with $\mu(\Gamma)=(-1)^{e(\Gamma)}$ denoting the Möbius function of the Boolean lattice $\mathcal{B}$ of all simple graphs on $1, \ldots, n)$ to the numbers

$$
\sharp\left(\mathcal{R}_{\Gamma}\right)=\sum_{\Gamma^{\prime} \supset \Gamma} \alpha_{\Gamma^{\prime}}
$$

given by Proposition 11.

\section{Proof of Proposition 5: Combinatorics of generic packings}

A hypergraph consists of a set $\mathcal{V}$ of vertices and of a set of hyperedges where a hyperedge is a subset of $\mathcal{V}$ containing at least 2 vertices. Two vertices are adjacent if they belong to a common hyperedge. A path is a sequence of consecutively adjacent vertices. A hypergraph is connected if any pair of vertices can be joined by a path. A cycle is a closed path involving only distinct vertices. A hyperforest is a hypergraph with distinct hyperedges intersecting in at most a common vertex and with every cycle contained in a hyperedge. A hypertree is a connected hyperforest.

The primal graph of a hypergraph with vertices $\mathcal{V}$ is the ordinary graph with vertices $\mathcal{V}$ and ordinary edges encoding adjacency in the hypergraph. An ordinary graph $\Gamma$ is the primal graph of a hyperforest if and only if every cycle and every edge of $\Gamma$ is contained in a unique maximal complete subgraph. Maximal complete subgraphs of such a graph $\Gamma$ 
are in one-to-one correspondence with hyperedges of the associated hyperforest. Primal graphs of hyperforests are often called block-graphs or chordal and diamond-free graphs. In the sequel, we identify generally hyperforests with their primal graphs.

Lemma 15. The intersection $g_{i} \mathcal{S}_{i} \cap g_{j} \mathcal{S}_{j}$ associated to an edge $\{i, j\}$ in an intersection graph $\mathcal{I}(\mathbf{g})$ is reduced to a unique element if $\mathcal{S}_{1}, \ldots, \mathcal{S}_{n}$ is a generic family of $G$.

Proof. Otherwise there exist two distinct elements $a_{i}, b_{i} \in \mathcal{S}_{j}$ and two distinct elements $a_{j}, b_{j} \in \mathcal{S}_{j}$ such that $g_{i} a_{i}=g_{j} b_{j}$ and $g_{j} a_{j}=g_{i} b_{i}$. This shows

$$
g_{i} a_{i} b_{j}^{-1} g_{j}^{-1} g_{j} a_{j} b_{i}^{-1} g_{i}^{-1}=e
$$

and implies the relation $b_{i}^{-1} a_{i} b_{j}^{-1} a_{j}=e$ with $b_{i}^{-1} a_{i} \in \mathcal{S}_{i}^{-1} \mathcal{S}_{i} \backslash\{e\}$ and $b_{j}^{-1} a_{j} \in \mathcal{S}_{j}^{-1} \mathcal{S}_{j} \backslash\{e\}$ in contradiction with genericity of the family $\mathcal{S}_{1}, \ldots, \mathcal{S}_{n}$.

Proposition 16. Intersection graphs of generic families are (primal graphs of) hyperforests.

Proof. Consider $k$ cyclically consecutive vertices $i_{1}, i_{2}, \ldots, i_{k-1}, i_{k}, i_{k+1}=i_{1}$ in an intersection graph $\mathcal{I}(\mathrm{g})$ of a generic family $\mathcal{S}_{1}, \ldots, \mathcal{S}_{n} \subset G$. Lemma 15 implies the existence of unique elements $a_{i_{j}} \in \mathcal{S}_{i_{j}}$ and $b_{i_{j+1}} \in \mathcal{S}_{i_{j+1}}$ such that $g_{i_{j}} a_{i_{j}}=g_{i_{j+1}} b_{i_{j+1}}$ for every edge $\left\{i_{j}, i_{j+1}\right\}$ of $C$. We get thus the relation

$$
g_{i_{1}} a_{i_{1}}\left(g_{i_{2}} b_{i_{2}}\right)^{-1} g_{i_{2}} a_{i_{2}}\left(g_{i_{3}} b_{i_{3}}\right)^{-1} \cdots g_{i_{k}} a_{i_{k}}\left(g_{i_{1}} b_{i_{1}}\right)^{-1}=e
$$

which is conjugate to the relation

$$
\left(b_{i_{1}}^{-1} a_{i_{1}}\right)\left(b_{i_{2}}^{-1} a_{i_{2}}\right) \cdots\left(b_{i_{k}}^{-1} a_{i_{k}}\right)=e .
$$

Genericity of the family $\mathcal{S}_{1}, \ldots, \mathcal{S}_{n}$ implies $a_{i_{j}}=b_{i_{j}}$ for all $j$. The sets $g_{i_{j}} \mathcal{S}_{i_{j}}$ intersect thus in the common element $g_{i_{1}} a_{i_{1}}=\cdots=g_{i_{k}} a_{i_{k}}$ (which is the unique common element of pairwise distinct sets in $\left\{g_{i_{1}} \mathcal{S}_{i_{1}}, \ldots, g_{i_{k}} \mathcal{S}_{i_{k}}\right\}$ by Lemma 15). All elements $i_{1}, \ldots, i_{k}$ of $\mathcal{I}(\mathbf{g})$ are thus adjacent vertices contained in a common maximal complete subgraph of $\mathcal{I}(\mathbf{g})$.

Suppose now that an edge $\{i, j\}$ belongs to two distinct maximal complete subgraphs $K$ and $K^{\prime}$ of $\mathcal{I}(\mathbf{g})$. Maximality of $K$ and $K^{\prime}$ implies the existence of vertices $k \in K \backslash K^{\prime}$ and $k^{\prime} \in K^{\prime} \backslash K$. Thus we get triplets of mutually adjacent vertices $i, j, k \subset K$ and $i, j, k^{\prime} \subset K^{\prime}$. Lemma 15 shows that $g_{i} \mathcal{S}_{i} \cap g_{j} \mathcal{S}_{j}$ is reduced to a unique element $a$. We have thus $g_{i} \mathcal{S}_{i} \cap g_{j} \mathcal{S}_{j} \cap g_{k} \mathcal{S}_{k}=\{a\} \subset K$. Similarly, we get $a \in g_{k^{\prime}} \mathcal{S}_{k^{\prime}}$. This implies $k^{\prime} \in K$ in contradiction with $k^{\prime} \in K^{\prime} \backslash K$.

Distinct maximal complete subgraphs of $\mathcal{I}(\mathbf{g})$ intersect thus at most in a common vertex and every cycle of $\mathcal{I}(\mathbf{g})$ is contained in a unique maximal complete subgraph of $\mathcal{I}(\mathbf{g})$. This implies that $\mathcal{I}(\mathbf{g})$ is (the primal graph of) a hyperforest.

For the sake of concision, we identify in the sequel such an intersection graph $\mathcal{I}(\mathbf{g})$ with the corresponding hyperforest.

Applying the proof of Proposition 16 to a Hamiltonian cycle visiting all vertices of a hyperedge $\left\{i_{1}, \ldots, i_{k}\right\}$ in an intersection graph $\mathcal{I}(\mathbf{g})$ associated to a generic family we get the following result: 
Proposition 17. Given a hyperedge $\left\{i_{1}, \ldots, i_{k}\right\}$ in the intersection graph $\mathcal{I}(\mathbf{g})$ of a generic family $\mathcal{S}_{1}, \ldots, \mathcal{S}_{n}$, there exists a unique element $a \in G$ such that $g_{i_{l}} \mathcal{S}_{i_{l}} \cap g_{i_{m}} \mathcal{S}_{i_{m}}=\{a\}$ for every pair of distinct vertices $i_{l}, i_{m}$ in $\left\{i_{1}, \ldots, i_{k}\right\}$.

Proposition 18. Let $\Gamma$ be a hyperforest with vertices $1, \ldots, n$ indexing the subsets $\mathcal{S}_{j}$ of a generic family in a group $G$. Defining the equivalence relation $\mathcal{E}_{F}$ of a hyperforest $F$ as in Section 3.2, we have

$$
\sharp\left(\mathcal{E}_{F}\right)=\prod_{j=1}^{n}\left(\sharp\left(\mathcal{S}_{j}\right)\right)^{\operatorname{deg}_{F}(j)}
$$

with $\operatorname{deg}_{F}(j)$ denoting the degree of $j$ defined as the number of distinct hyperedges containing the vertex $j$.

Proof. Let $e=\left\{i_{1}, \ldots, i_{k}\right\}$ be a hyperedge of an intersection graph $\mathcal{I}(\mathbf{g})$. Since $\bigcap_{j=1}^{k} g_{i_{j}} \mathcal{S}_{i_{j}}$ is reduced to a unique element $a_{e} \in G$, we get a map $\mu_{e}:\left\{i_{1}, \ldots, i_{k}\right\} \longrightarrow G$ such that $\mu_{e}\left(i_{j}\right) \in \mathcal{S}_{i_{j}}$ by setting $\mu_{e}\left(i_{j}\right)=g_{i_{j}}^{-1} a_{e}$. This map depends only on the equivalence class in $\mathcal{E}_{\mathcal{I}(\mathbf{g})}$ of $\mathcal{I}(\mathbf{g})$ and the set of all such maps determines the equivalence class of $\mathcal{I}(\mathbf{g})$ in $\mathcal{E}_{F}$ for any hyperforest $F$ contained in $\mathcal{I}(\mathbf{g})$. Since all cycles of a hyperforest are contained in hyperedges, all possible choices of the maps $\mu_{e}$ associated to hyperedges of $F$ correspond to equivalence classes of $\mathcal{E}_{F}$. Different choices yield inequivalent classes. The set $\mathcal{E}_{F}$ of all equivalence classes is thus in one-to-one correspondence with the set $\prod_{j=1}^{n} \mathcal{S}_{j}^{\operatorname{deg}_{F}(j)}$.

Proof of Proposition 5. Setting $s_{i}=\sharp\left(\mathcal{S}_{i}\right)$, Proposition 18 can be rewritten as the identity

$$
\sharp\left(\mathcal{E}_{F}\right)=\prod_{j=1} s_{j}^{\operatorname{deg}_{F}(j)}
$$

for every hyperforest $F$ with vertices $\{1, \ldots, n\}$. We denote by $\mathcal{H} \mathcal{F}(n)$ the set of all hyperforests with vertices $\{1, \ldots, n\}$. The set $\mathcal{H} \mathcal{F}(n)$ is partially ordered by inclusion by setting $F^{\prime} \leqslant F$ for $F^{\prime}, F \in \mathcal{H} \mathcal{F}(n)$ if every hyperedge of $F^{\prime}$ is contained in some hyperedge of $F$. Equivalently, $F^{\prime} \leqslant F$ if adjacent vertices of $F^{\prime}$ are also always adjacent in $F$. The primal graph underlying $F^{\prime}$ is thus a subgraph of the primal graph underlying $F$ if $F^{\prime} \leqslant F$. Denoting by $\mu$ the Möbius function of the poset $\mathcal{H} \mathcal{F}(n)$, the number $\alpha=\alpha\left(G ; \mathcal{S}_{1}, \ldots, \mathcal{S}_{n}\right)$ of packings of a generic family $\mathcal{S}_{1}, \ldots, \mathcal{S}_{n}$ in a group $G$ of order $N$ is given by

$$
\alpha=\sum_{F \in \mathcal{H} \mathcal{F}(n)} \mu(F) N^{c(F)} \prod_{j=1}^{n} s_{j}^{\operatorname{deg}_{F}(j)}
$$

(with $c(F)$ denoting the number of connected components of a hyperforest $F$ ). Since the Möbius function of $\mathcal{H} \mathcal{F}(n+1)$ restricts to the Möbius function of $\mathcal{H} \mathcal{F}(n)$, the summation over $\mathcal{H} \mathcal{F}(n)$ in Formula 8 can be extended (after setting $s_{i}=0$ for $i>n$ and using the convention $0^{0}=1$ ) over the poset $\mathcal{H} \mathcal{F}$ of all hyperforests with vertices $\mathbb{N} \backslash\{0\}$ such that almost all vertices are isolated (only finitely many vertices have strictly positive degree).

Summing over all possible labellings of an unlabelled hyperforest and remarking that the Möbius function is invariant under permutations of labels shows that $\alpha$ is a symmetric 
function of $s_{1}, \ldots, s_{n}$. This expresses $\alpha$ as a polynomial in $\sigma_{1}=\sum s_{i}, \sigma_{2}=\sum_{i<j} s_{i} s_{j}, \ldots$ with contributions coming from finite unlabelled hyperforests.

More precisely, contributions to the coefficient $N^{n-m}$ of $\alpha$ given by Formula 8 come from hyperforests with vertices $\{1, \ldots, n\}$ consisting of $c \geqslant 0$ non-trivial hypertrees involving $m+c \leqslant n$ vertices of strictly positive degrees and $n-m-c$ isolated vertices. The equalities $s_{n+1}=s_{n+2}=\cdots=0$ imply that the summation over $\mathcal{H} \mathcal{F}(n)$ in 8 can be extended to a summation over all hyperforests in $\mathcal{H} \mathcal{F}(l)$ for an arbitrary integer $l \geqslant n$ since the vertices $n+1, n+2, \ldots$ have to be isolated vertices of a hyperforest yielding a non-zero contribution to $\alpha$. Observe now that we have $c \leqslant m$ since every non-trivial hypertree contains at least two vertices. For a fixed value of $m$, a labelled hyperforest with non-zero contribution to $\alpha$ has thus at most $2 m$ non-isolated vertices (with equality achieved by a hyperforest consisting of $m$ isolated edges joining $2 m$ distinct vertices). Summing over unlabelled hyperforests and considering the associated symmetric functions $\sigma_{1}, \sigma_{2}, \ldots$ in $s_{1}, s_{2}, \ldots$ (obtained by a summation over all possible distinct labellings of the underlying unlabelled hypertrees) we see that the contribution associated to an unlabelled hyperforest with $m+c \leqslant 2 m$ non-isolated vertices is in the ideal of $\mathbb{Z}\left[x, \sigma_{1}, \sigma_{2}, \ldots\right]$ generated by $\sigma_{m+c}, \sigma_{m+c+1}, \sigma_{m+c+2}, \ldots$ The degree in $\sigma_{1}, \sigma_{2}, \ldots$ (with respect to the grading $\operatorname{deg}\left(\sigma_{i}\right)=i$ ) of such a contribution is maximal and equals $2 m$ for ordinary unlabelled forests having $m$ ordinary edges. Indeed, let $F$ be a hyperforest with $c$ connected components and $m+c$ vertices of strictly positive degrees. Replacing a hyperedge $E$ of $F$ involving $k \geqslant 3$ vertices by a tree consisting of $k-1$ ordinary edges connecting all vertices of $E$ increases the degree-sum of all vertices by $k-2>0$ and yields a contribution of higher degree. Contributions of maximal degree correspond thus to ordinary forests on $m+c$ vertices with $c$ connected components. Such a forest has $m$ edges and yields a contribution of degree $2 m$ with respect to the grading $\operatorname{deg}\left(\sigma_{i}\right)=i$. This ends the proof of Proposition 5.

\section{$5 \quad$ Proof of Proposition 7}

Proof of Proposition 7. We have to show that

$$
U=1-\sum_{n=1}^{\infty} x^{n} \sum_{i=n+1}^{2 n} \sigma_{i} \sum_{j=0}^{2 n-i} t_{i, j}(n)\left(-\sigma_{1}\right)^{j}
$$

defined by Formula 4 satisfies the functional equation

$$
\left(1-\sigma_{1} x\right) U\left(x, \sigma_{1}, \sigma_{2}, \sigma_{3}, \ldots\right)=U\left(x, 1+\sigma_{1}, \sigma_{1}+\sigma_{2}, \sigma_{2}+\sigma_{3}, \ldots\right),
$$

see 6 . Both sides of 6 have the same constant term 1 and involve only non-constant monomials of the form $\sigma_{i} \sigma_{1}^{j} x^{n}$. It is thus enough to check that coefficients of both sides of 6 agree for such monomials. This is easily checked for the coefficient of $x$. For a general 
monomial of the form $\sigma_{i} \sigma_{1}^{j} x^{n}$, equation 6 amounts to the identity

$$
\begin{aligned}
& -\left((-1)^{j} t_{i, j}(n)-(-1)^{j-1} t_{i, j-1}(n-1)\right) \\
= & -\left(\sum_{k=j}^{2 n-i} t_{i, k}(n)(-1)^{k}\left(\begin{array}{l}
k \\
j
\end{array}\right)+\sum_{k=j}^{2 n-i-1} t_{i+1, k}(n)(-1)^{k}\left(\begin{array}{l}
k \\
j
\end{array}\right)\right)
\end{aligned}
$$

or equivalently to

$$
t_{i, j}(n)+t_{i, j-1}(n-1)=\sum_{k}(-1)^{k+j}\left(\begin{array}{l}
k \\
j
\end{array}\right)\left(t_{i, k}(n)+t_{i+1, k}(n)\right)
$$

where $\sum_{k} f(k)=\sum_{k \in \mathbb{Z}} f(k)$ since $\left(\begin{array}{l}k \\ j\end{array}\right)\left(t_{i, k}(n)+t_{i+1, k}(n)\right)=0$ for $k<j$ or $k>2 n-i$. We prove 9 by induction on $n$. A straightforward computation shows that it holds $n=2$. Applying the recursion relation 2 which holds for all $i, j \in \mathbb{Z}$ if $n \geqslant 2$ to the right side

$$
R=\sum_{k}(-1)^{k+j}\left(\begin{array}{l}
k \\
j
\end{array}\right)\left(t_{i, k}(n)+t_{i+1, k}(n)\right)
$$

of 9 we get

$$
\begin{aligned}
R= & \sum_{\substack{k \\
(i-1)}}(-2) t_{i-1, k}(n-1)+t_{i-1, k-1}(n-1)+(i-3) t_{i-2, k}(n-1) \\
& \left.\quad+(i-1) t_{i, k}(n-1)+t_{i, k-1}(n-1)+(i-2) t_{i-1, k}(n-1)\right) \\
& \quad+\quad+C
\end{aligned}
$$

where

$$
\begin{aligned}
L=(i & -2) \sum_{k}(-1)^{k+j}\left(\begin{array}{c}
k \\
j
\end{array}\right)\left(t_{i-1, k}(n-1)+t_{i, k}(n-1)\right) \\
& +\sum_{k}(-1)^{k+j-1}\left(\begin{array}{c}
k \\
j-1
\end{array}\right)\left(t_{i-1, k}(n-1)+t_{i, k}(n-1)\right) \\
& +(i-3) \sum_{k}(-1)^{k+j}\left(\begin{array}{c}
k \\
j
\end{array}\right)\left(t_{i-2, k}(n-1)+t_{i-1, k}(n-1)\right)
\end{aligned}
$$


and

$$
\begin{aligned}
C=- & \sum_{k}(-1)^{k+j-1}\left(\begin{array}{c}
k \\
j-1
\end{array}\right)\left(t_{i-1, k}(n-1)+t_{i, k}(n-1)\right) \\
& +\sum_{k}(-1)^{k+j}\left(\begin{array}{c}
k \\
j
\end{array}\right)\left(t_{i-1, k-1}(n-1)+t_{i, k-1}(n-1)\right) \\
& +\sum_{k}(-1)^{k+j}\left(\begin{array}{c}
k \\
j
\end{array}\right)\left(t_{i, k}(n-1)+t_{i-1, k}(n-1)\right) \\
= & \sum_{k}(-1)^{k+j}\left(\begin{array}{c}
k \\
j-1
\end{array}\right)\left(t_{i-1, k}(n-1)+t_{i, k}(n-1)\right) \\
& -\sum_{k}(-1)^{k+j}\left(\begin{array}{c}
k+1 \\
j
\end{array}\right)\left(t_{i-1, k}(n-1)+t_{i, k}(n-1)\right) \\
& +\sum_{k}(-1)^{k+j}\left(\begin{array}{c}
k \\
j
\end{array}\right)\left(t_{i-1, k}(n-1)+t_{i, k}(n-1)\right) \\
= & \sum_{k}(-1)^{k+j}\left(\left(\begin{array}{c}
k \\
j-1
\end{array}\right)-\left(\begin{array}{c}
k+1 \\
j
\end{array}\right)+\left(\begin{array}{c}
k \\
j
\end{array}\right)\right)\left(t_{i-1, k}(n-1)+t_{i, k}(n-1)\right)
\end{aligned}
$$

which shows $C=0$ since $\left(\begin{array}{c}k+1 \\ j\end{array}\right)=\left(\begin{array}{c}k \\ j-1\end{array}\right)+\left(\begin{array}{c}k \\ j\end{array}\right)$.

Using induction on $n$ and applying 9 we get

$$
\begin{aligned}
L=(i-2)\left(t_{i-1, j}(n-1)+t_{i-1, j-1}(n-2)\right) \\
\quad+\left(t_{i-1, j-1}(n-1)+t_{i-1, j-2}(n-2)\right) \\
\quad+(i-3)\left(t_{i-2, j}(n-1)+t_{i-2, j-1}(n-2)\right)
\end{aligned}
$$

We have thus

$$
\begin{aligned}
L= & (i-2) t_{i-1, j}(n-1)+t_{i-1, j-1}(n-1)+(i-3) t_{i-2, j}(n-1) \\
& +(i-2) t_{i-1, j-1}(n-2)+t_{i-1, j-2}(n-2)+(i-3) t_{i-2, j-1}(n-2)
\end{aligned}
$$

and applying 2 we get

$$
L=t_{i, j}(n)+t_{i, j-1}(n-1)
$$

which is the left side of 9 .

\section{Proof of Proposition 8}

Proof of Proposition 8. Assuming the existence of two distinct series $U_{1}, U_{2}$ fulfilling the requirements of Proposition 8, the difference $D=U_{1}-U_{2}=\sum_{n=1}^{\infty} D_{n} x^{n}$ satisfies all hypotheses except for the value of its constant term. Since $U_{1}$ and $U_{2}$ are different, there exists a minimal natural integer $n \geqslant 1$ such that $D_{n} \neq 0$. Let $m \geqslant n+1$ be the smallest integer such that $D_{n}=\sum_{k=m}^{2 n} \sigma_{k} C_{n, k}$ with $C_{n, k} \in \mathbb{C}\left[\sigma_{1}, \sigma_{2}, \ldots\right]$ and $C_{n, m} \neq 0$. 
Since $D_{n}$ is of degree $\leqslant 2 n$ with respect to the grading given by $\operatorname{deg}\left(\sigma_{i}\right)=i$, we have $C_{n, m} \in \mathbb{C}\left[\sigma_{1}, \ldots, \sigma_{2 n-m}\right] \subset \mathbb{C}\left[\sigma_{1}, \ldots, \sigma_{n-1}\right]$.

Equation 6 and minimality of $n$ imply

$$
D_{n}\left(1+\sigma_{1}, \sigma_{1}+\sigma_{2}, \sigma_{2}+\sigma_{3}, \ldots\right)=D_{n}\left(\sigma_{1}, \sigma_{2}, \sigma_{3}, \ldots\right)
$$

or equivalently

$$
\sum_{k=m}^{2 n}\left(\sigma_{k-1}+\sigma_{k}\right) C_{n, k}\left(1+\sigma_{1}, \sigma_{1}+\sigma_{2}, \sigma_{2}+\sigma_{3}, \ldots\right)=\sum_{k=m}^{2 n} \sigma_{k} C_{n, k}\left(\sigma_{1}, \sigma_{2}, \sigma_{3}, \ldots\right) .
$$

Comparison of both sides modulo the ideal $I$ generated by $\sigma_{m}, \sigma_{m+1}, \sigma_{m+2}, \ldots$ gives

$$
C_{n, m}\left(1+\sigma_{1}, \sigma_{1}+\sigma_{2}, \sigma_{2}+\sigma_{3}, \ldots\right)=0 .
$$

Algebraic independency of the symmetric functions $\sigma_{1}, \sigma_{2}, \ldots$ shows thus $C_{n, m}=0$ in contradiction with our assumption.

\section{The Möbius function for the poset of finite labelled hyperforests}

Let $\mathcal{P}$ be a poset (partially ordered set) such that $\mathcal{P}$ has a unique minimal element $m$ and $\{y \in \mathcal{P} \mid y<x\}$ is finite for all $x \in \mathcal{P}$. This allows the recursive definition of a Möbius function $\mu$ by setting $\mu(m)=1$ and $\mu(x)=-\sum_{y<x} \mu(y)$ for all $x>m$. Given a function $f: \mathcal{P} \longrightarrow \mathbb{C}$ with finite support, the value $f(m)$ can then be recovered from the function $g(x)=\sum_{y \geqslant x} f(y)$ using Möbius inversion

$$
f(m)=\sum_{x \in \mathcal{P}} \mu(x) g(x)
$$

see Proposition 3.7.2 of [5] (we use only the values $\mu(m, x)$ of the Möbius function and write $\mu(x)=\mu(m, x)$ in analogy with the usual, well-known number-theoretic Möbius function of natural integers). Möbius inversion was the main ingredient in the proof of Proposition 5. The poset $\mathcal{H} \mathcal{F}$ of hyperforests consisting of all hyperforests (ordered by inclusion) with finitely many hyperedges and vertices $1,2,3,4, \ldots$ has a minimal element given by the trivial graph having only isolated vertices. The number

$$
\sharp\left\{F^{\prime} \in \mathcal{H} \mathcal{F} \mid F^{\prime} \subset F\right\}
$$

of all hyperforests contained in a given hyperforest $F \in \mathcal{H} \mathcal{F}$ with $n$ hyperedges of degrees $d_{1}, \ldots, d_{n}$ is bounded by the number $2\left(\begin{array}{c}\left(\begin{array}{c}d_{1} \\ 2\end{array}\right)+\cdots+\left(\begin{array}{c}d_{n} \\ 2\end{array}\right) \\ \text { of (ordinary) subgraphs of the primal }\end{array}\right.$ graph underlying $F$. The poset $\mathcal{H} \mathcal{F}$ has thus a Möbius function. 
Proposition 19. The Möbius function $\mu(F)$ of a hyperforest $F$ in the poset $\mathcal{H} \mathcal{F}$ of all vertex-labelled hyperforests with finitely many hyperedges is given by

$$
\mu(F)=\prod_{j \geqslant 2}(-(j-2) !)^{\kappa_{j}}
$$

where $\kappa_{j}$ denotes the number of hyperedges involving exactly $j$ vertices of $F$.

Remark 20. The poset $\mathcal{H} \mathcal{F}$ is in fact a lattice with wedge $F_{1} \wedge F_{2}$ given by the intersection and join $F_{1} \vee F_{2}$ given by the smallest hyperforest containing $F_{1}$ and $F_{2}$.

Proof of Proposition 19. Remark first that the order relation induced on subforests of a given hyperforest $F \in \mathcal{H} \mathcal{F}$ is the product order of all order-relations on hyperedges of $F$. An easy argument (or Proposition 3.8.2 of [5]) shows thus that we have

$$
\mu(F)=\prod_{e \in E(F)} \mu(e)
$$

where $E(F)$ denotes the set of hyperedges of $F$ and where $\mu(e)$ is the Möbius function restricted to a hyperedge $e \in E(F)$. This can of course be rewritten as

$$
\mu(F)=\prod_{j \geqslant 2} \mu\left(K_{j}\right)^{\kappa_{j}}
$$

where $K_{j}$ is an abitrary hyperedge on $j$ labelled vertices and where $\kappa_{j}$ is the number of hyperedges having $j$ vertices of $F$.

The proof of Proposition 5 shows that $\mu\left(K_{j}\right)$ coincides with the coefficient of $\sigma_{j+1} x^{j}$ in $U$. By Theorem 4 (whose proof needs only the existence but not the exact determination of the Möbius function), this coefficient equals $-t_{j+1,0}(j)=-(j-2)$ ! where the last identity follows easily from Formula 2 defining the integers $t_{i, j}(n)$ recursively.

Remark 21. It would be interesting to have a simple direct proof that $\mu\left(K_{n}\right)=-(n-2)$ ! for a hypergraph $K_{n} \in \mathcal{H} \mathcal{F}$ reduced to a unique hyperedge involving $n \geqslant 2$ vertices.

Remark 22. Let $\mathcal{H} \mathcal{T}_{k}(n)$ be the finite set of all hypertrees with $k$ hyperedges and $n$ labelled vertices. Denoting by $\sharp(e)$ the number of vertices involved in a hyperedge $e$, we have

$$
\sum_{T \in \mathcal{H} \mathcal{T}_{k}(n)} \prod_{e \in \mathcal{E}(T)}(\sharp(e)-2) ! \prod_{j=1}^{n} s_{j}^{\operatorname{deg}(j)}=(-1)^{n+k+1} \sigma_{n} \sigma_{1}^{k-1} S_{1}(n-1, k)
$$

where $\sigma_{1}=\sum_{j=1}^{n} s_{j}$ and $\sigma_{n}=\prod_{j=1}^{n} s_{j}$ and where $S_{1}(n, k)$ denotes the Stirling number of the first kind defined by

$$
\sum_{k=0}^{n} S_{1}(n, k) x^{k}=x(x-1)(x-2) \cdots(x-n+1)=\prod_{j=0}^{n-1}(x-j) .
$$


Indeed, the proof of Proposition 5 shows that a hyperforest with $n$ non-isolated vertices, $k$ hyperedges and $c$ connected components yields only contributions to the coefficients of $x^{n-c} \sigma_{n+s} \sigma_{1}^{k-1-s}$ for $s=0, \ldots, k-1$. The coefficient of $x^{n-1} \sigma_{n} \sigma_{1}^{k-1}$ in $U$ is thus obtained from contributions from all elements in the set $\mathcal{H} \mathcal{T}_{k}(n)$ of hypertrees with $n$ non-isolated vertices $\{1, \ldots, n\}$ and $k$ hyperedges. This coefficient equals $(-1)^{n+1} \sigma_{n} \sigma_{1}^{k-1} S_{1}(n-1, k)$ by Formulae 4 and 3. Formulae 8 and 10 show that a hypertree $T \in \mathcal{H} \mathcal{T}_{k}(n)$ contributes a summand given by $(-1)^{k} \prod_{e \in \mathcal{E}(T)}(\sharp(e)-2) ! \prod_{j=1}^{n} s_{j}^{\operatorname{deg}(j)}$ to the coefficient of $x^{n-1} \sigma_{n} \sigma_{1}^{k-1}$ in $U$.

Setting $s_{1}=\cdots=s_{n}=1$, Formula 11 specializes to the identity

$$
\sum_{T \in \mathcal{H} \mathcal{T}_{k}(n)} \prod_{e \in \mathcal{E}(T)}(\sharp(e)-2) !=n^{k-1} S_{1}(n-1, k)(-1)^{n+k+1}
$$

which is analogous to a Theorem of Husimi (see [2] or [1]) expressing the total number

$$
n^{k-1} S_{2}(n-1, k)
$$

of elements in the set $\mathcal{H} \mathcal{T}_{k}(n)$ of labelled hypertrees with $k$ hyperedges and $n$ vertices in terms of Stirling numbers of the second kind.

All these results are of course generalizations and variations of Cayley's theorem corresponding to the case $k=n-1$ and showing that there are $n^{n-2}$ labelled trees on $n$ vertices.

Observe that all these identities can also be deduced for example from Exercice 5.30 in [5] using a well-known map between hypergraphs and ordinary bipartite graphs.

\section{Computational aspects and examples}

The computation of $U\left(x, \sigma_{1}, \sigma_{2}, \ldots\right)$ up to $o\left(x^{n}\right)$ is straightforward using the recurrence relation 2. For a given fixed numerical value of $\sigma_{1}$, the following trick reduces memory requirement and speeds the computation up: Setting

$$
c_{n}\left(\sigma_{1}\right)=\left(\gamma_{n+1}\left(\sigma_{1}, n\right), \gamma_{n+2}\left(\sigma_{1}, n\right), \ldots, \gamma_{2 n}\left(\sigma_{1}, n\right)\right)
$$

with $\gamma_{i}\left(\sigma_{1}, n\right)=\sum_{j=0}^{2 n-i} t_{i, j}(n)\left(-\sigma_{1}\right)^{j}$ we have

$$
U\left(x, \sigma_{1}, \sigma_{2}, \ldots\right)=1-\sum_{n=1}^{\infty}\left\langle c_{n}\left(\sigma_{1}\right),\left(\sigma_{n+1}, \ldots, \sigma_{2 n}\right)\right\rangle x^{n}
$$

where $\langle a, b\rangle=\sum_{i \in I} a_{i} b_{i}$ for two finite-dimensional vectors $a, b$ with coefficients indexed by a common finite set $I$. The coefficients $\gamma_{i}\left(\sigma_{1}, n\right)$ of $c_{n}\left(\sigma_{1}\right)$ can be computed from the coefficients of $c_{n-1}\left(\sigma_{1}\right)$ by the formula

$$
\gamma_{i}\left(\sigma_{1}, n\right)=\left(i-2-\sigma_{1}\right) \gamma_{i-1}\left(\sigma_{1}, n-1\right)+(i-3) \gamma_{i-2}\left(\sigma_{1}, n-1\right)
$$

with missing coefficients omitted in the case of $i=n+1$ or $i=2 n$. 
The coefficients of the first vectors $c_{1}(0), c_{2}(0), c_{3}(0), \ldots$ are given by the rows of

$\begin{array}{rrrrr}1 & & & & \\ 1 & 1 & & & \\ 2 & 5 & 3 & & \\ 6 & 26 & 35 & 15 & \\ 24 & 154 & 340 & 315 & 105\end{array}$

see A112486 of [4].

\subsection{The examples $U(x,-1,-1,-1, \ldots)$ and $U(x, 0,-1,-1,-1, \ldots)$}

The series

$$
U(x,-1,-1,-1,-1, \ldots)-1
$$

is the generating series of the sequence

$$
S(n)=\sum_{i, j} t_{i, j}(n)
$$

enumerating the sums of the triangles $T(n)$ defined by the integers $t_{i, j}(n)$. We have

$$
\begin{aligned}
& (1+x) U(x,-1,-1,-1,-1, \ldots) \\
= & U(x, 0,-2,-2,-2,-2, \ldots) \\
= & 2 U(x, 0,-1,-1,-1,-1, \ldots)-1
\end{aligned}
$$

where $U(x, 0,-1,-1,-1, \ldots)-1$ corresponds to the generating series of the sequence

$$
s(n)=\sum_{i=n+1}^{2 n} t_{i, 0}(n)
$$

starting as

$$
1,2,10,82,938,13778,247210,5240338,128149802,3551246162, \ldots,
$$

cf. A112487 of [4], and obtained by summing the integers of the first column of the triangles $T(1), T(2), \ldots$ In particular, we have $2 s(n)=S(n-1)+S(n)$ or equivalently

$$
2 \sum_{i=n+1}^{2 n} t_{i, 0}(n)=\sum_{i=n+1}^{2 n} \sum_{j=0}^{2 n-i} t_{i, j}(n)+\sum_{i=n}^{2 n-2} \sum_{j=0}^{2 n-2-i} t_{i, j}(n-1)
$$

for all $n \geqslant 2$. 


\subsection{Examples satisfying differential equations}

The recursive definition of the integers $t_{i, j}(n)$ implies easily that specializations of the form

$$
\sigma_{n}=c \frac{\prod_{k=1}^{A}\left(n+a_{k}\right) !}{\prod_{l=1}^{B}\left(n+b_{l}\right) !} z^{\lambda n+r}, n \geqslant 2,
$$

or

$$
\sigma_{n}=c \frac{\prod_{k=1}^{A}\left(n+a_{k}\right) !}{\prod_{l=1}^{B}\left(n+b_{l}\right) !} e^{(\lambda n+r) z}, n \geqslant 2,
$$

(with $\lambda \neq 0$ and $b_{i} \notin\{-2,-3,-4,-5, \ldots\}$ ) lead to differential equations with respect to $z$ for rational expressions of $U\left(x,-y, \sigma_{2}, \sigma_{3}, \sigma_{4}, \ldots\right)$. Such a series $U$ is analytic if $B>A$. We illustrate this with the following examples.

\subsubsection{U(x,-y,- $\left.z^{2+r},-z^{3+r},-z^{4+r}, \ldots\right)$}

Setting $\sigma_{n}=-z^{n+r}$ for $n=2,3, \ldots$ the series $f(z)=U\left(x,-y, \sigma_{2}, \sigma_{3}, \ldots\right)-1$ satisfies formally the differential equation

$$
f=x z\left(z^{r+1}+(y-(1+z)(1+r)) f+z(1+z) \frac{d f}{d z}\right) .
$$

8.2.2 U $U\left(x,-y,-\frac{z^{2+r}}{(2+b) !},-\frac{z^{3+r}}{(3+b) !},-\frac{z^{4+r}}{(4+b) !}, \ldots\right)$

Setting $\sigma_{n}=-\frac{z^{n+r}}{(n+b) !}$ for $n=2,3, \ldots$ the series $f(z)=U\left(x,-y, \sigma_{2}, \sigma_{3}, \ldots\right)-1$ satisfies the differential equation

$$
\begin{aligned}
& (b-r)(b-1-r) f+2(b-r) z f^{\prime}+z^{2} f^{\prime \prime} \\
= & \frac{x z^{2+r}}{b !}+x z(r-b-z(r+1)+(r-b)(r-y)) f \\
+ & x z^{2}(b+y+z-2 r) f^{\prime}+x z^{3} f^{\prime \prime}
\end{aligned}
$$

8.2.3U $U\left(x,-y,-e^{(2 \lambda+r) z},-e^{(3 \lambda+r) z},-e^{(4 \lambda+r) z}, \ldots\right)$

Setting $\sigma_{n}=-e^{(n \lambda+r) z}$ for $n=2,3, \ldots$, the series

$$
f(z)=U\left(x,-y, \sigma_{2}, \sigma_{3}, \sigma_{4}, \ldots\right)-1
$$

satisfies formally the differential equation

$$
f=x e^{\lambda z}\left(e^{(\lambda+r) z}+\left(y-\left(1+\frac{r}{\lambda}\right)\left(1+e^{\lambda z}\right)\right) f+\frac{1+e^{\lambda z}}{\lambda} \frac{d f}{d z}\right) .
$$


8.2.4U $U\left(x,-y,-(2+a) ! e^{(2 \lambda+r) z},-(3+a) ! e^{(3 \lambda+r) z},-(4+a !) e^{(4 \lambda+r) z}, \ldots\right)$

Setting $\sigma_{n}=-(n+a) ! e^{(n \lambda+r) z}$ for $n=2,3, \ldots$, the series $f(z)=U\left(x,-y, \sigma_{2}, \sigma_{3}, \ldots\right)-1$ satisfies formally the differential equation

$$
\begin{aligned}
f= & \frac{x e^{\lambda z}}{\lambda^{3}}(\lambda(1+a)-r)\left(\lambda(\lambda(y-1)-r)+(\lambda+r)(r-\lambda(2+a)) e^{\lambda z}\right) f \\
& +\frac{x e^{\lambda z}}{\lambda^{3}}\left(\lambda(\lambda(y+a)-2 r)+\left(3 r^{2}-4 \lambda r(a+1)+\lambda^{2}\left(a^{2}+a-1\right)\right) e^{\lambda z}\right) f^{\prime} \\
& +\frac{x e^{\lambda z}}{\lambda^{3}}\left(\lambda+(2 \lambda(1+a)-3 r) e^{\lambda z}\right) f^{\prime \prime}+\frac{x e^{2 \lambda z}}{\lambda^{3}} f^{\prime \prime \prime}+(2+a) ! x e^{(2 \lambda+r) z}
\end{aligned}
$$

8.2.5 U $\left(x,-y,-\frac{e^{(2 \lambda+r) z}}{(2+b) !},-\frac{e^{(3 \lambda+r) z}}{(3+b) !},-\frac{e^{(4 \lambda+r) z}}{(4+b) !} \cdots\right)$

Setting $\sigma_{n}=-\frac{e^{(n \lambda+r) z}}{(n+b) !}$ for $n=2,3, \ldots$ the series $f(z)=U\left(x,-y, \sigma_{2}, \sigma_{3}, \ldots\right)-1$ satisfies the differential equation

$$
\begin{aligned}
& (\lambda b-r)(\lambda(b-1)-r) f+(\lambda(2 b-1)-2 r) f^{\prime}+f^{\prime \prime} \\
= & x \frac{\lambda^{2}}{b !} e^{(2 \lambda+r) z}+x e^{\lambda z}\left((\lambda b-r)(\lambda(y-1)-r)-\lambda(\lambda+r) e^{\lambda z}\right) f \\
+ & x e^{\lambda z}\left(\lambda(b-1+y)-2 r+\lambda e^{\lambda z}\right) f^{\prime}+x e^{\lambda z} f^{\prime \prime}
\end{aligned}
$$

Remark 23. The recursion relation 2 gives rise to partial differential equations for generating series of $t_{i, j}(n)$ which are exponential with respect to $j$ and/or $n$.

\subsection{A family of rational examples}

Proposition 24. Let $\sigma_{1}, \sigma_{2}, \ldots$ be a sequence of complex numbers of the form $\sigma_{n}=$ $(-1)^{n} P(n)$ for all $n \geqslant A$ where $A$ is some natural integer and where $P(s) \in \mathbb{C}[s]$ is a polynomial. Then $U\left(x, \sigma_{1}, \sigma_{2}, \ldots\right)$ is a rational series.

Proof. Let $d$ denote the degree of $P$. Applying identity 6 of Proposition 6 iteratively $d+1$ times we get a series of the form $U\left(x, \tilde{\sigma}_{1}, \tilde{\sigma}_{2}, \ldots, \tilde{\sigma}_{A+d+2}, 0,0,0, \ldots\right)$ which is a polynomial.

As an illustration we consider the series $U(x, y, 1,-1,1, \ldots)$. Proposition 6 shows

$$
(1-x y) U(x, y, 1,-1,1,-1, \ldots)=U(x, 1+y, 1+y, 0,0, \ldots)=1-(1+y) x .
$$

We have thus $U(x, y, 1,-1,1, \ldots)=1-\frac{x}{1-x y}$. 


\subsection{Coefficients of $U\left(x, \sigma_{1}, P(2), P(3), P(4), \ldots\right)$}

Proposition 25. Let $P(s) \in \mathbb{C}[s]$ be a polynomial of degree $d$. There exist constants $\alpha_{0}, \ldots, \alpha_{d} \in \mathbb{C}$ such that

$$
\left[x^{n}\right] U\left(x, \sigma_{1}, P(2), P(3), P(4), \ldots\right)=\sum_{h=0}^{d} \alpha_{h}\left[x^{n+h}\right] U\left(x, \sigma_{1}, 1,1,1,1,1, \ldots\right)
$$

for all $n \geqslant 1$ with $\left[x^{n}\right] U$ denoting the coefficient of $x^{n}$ in the series $U$.

Proof. The proof is by induction on $d$ and holds certainly for $d=0$. Setting $\gamma_{i}(n)=$ $\sum_{j=n+1}^{2 n} t_{i, j}(n)\left(-\sigma_{1}\right)^{j}$, formula (12) implies

$$
\begin{aligned}
0= & -i^{d} \gamma_{i}(n+1)+i^{d}\left(i-2-\sigma_{1}\right) \gamma_{i-1}(n)+i^{d}(i-3) \gamma_{i-2}(n) \\
= & -i^{d} \gamma_{i}(n+1)+(i-1)^{d+1} \gamma_{i-1}(n)+(i-2)^{d+1} \gamma_{i-2}(n)+ \\
& +Q_{1}(i-1) \gamma_{i-1}(n)+Q_{2}(i-2) \gamma_{i-2}(n)
\end{aligned}
$$

where $Q_{1}$ and $Q_{2}$ are polynomials of degree $\leqslant d$. Fixing $n$ and summing over $i$ we get

$$
2 \sum_{i=n+1}^{2 n} i^{d+1} \gamma_{i}(n)=\sum_{i=n+2}^{2 n+2} i^{d} \gamma_{i}(n+1)-\sum_{i=n+1}^{2 n}\left(Q_{1}+Q_{2}\right)(i) \gamma_{i}(n) .
$$

The right side of 13 equals now

$$
\left[x^{n+1}\right] U\left(x, \sigma_{1}, 2^{d}, 3^{d}, 4^{d}, \ldots\right)-\left[x^{n}\right] U\left(x, \sigma_{1},\left(Q_{1}+Q_{2}\right)(2),\left(Q_{1}+Q_{2}\right)(3), \ldots\right) .
$$

It is thus by induction on $d$ a linear combination of the coefficients of $x^{n}, \ldots, x^{n+d+1}$ in $U\left(x, \sigma_{1}, 1,1,1, \ldots\right)$. This proves the result for $U\left(x, \sigma_{1}, 2^{d+1}, 3^{d+1}, \ldots\right)$. The general induction step follows by remarking that all coefficients of strictly positive degree in $x$ of $U\left(x, \sigma_{1}, \sigma_{2}, \ldots\right)$ are linear in $\sigma_{2}, \sigma_{3}, \ldots$

\section{Conjectural asymptotics for $s(1), s(2), \ldots$}

Computations with a few thousand values of $s(n)$ suggest the following asymptotic formula for the integral sequence $s(n)=\sum_{i=n+1}^{2 n} t_{i, 0}(n)$ :

Conjecture 26. There exists a sequence $A_{0}, A_{1}, \ldots$ of rational polynomials $A_{i}(x) \in \mathbb{Q}[x]$ with $A_{i}$ of degree $i$ such that

$$
s(n)=\frac{n^{n-1}}{(1-\log 2)^{n-1 / 2} e^{n}}\left(\sum_{k=0}^{m} \frac{A_{k}(1-\log 2)}{n^{k}}+o\left(n^{-m}\right)\right)
$$

for all $m \in \mathbb{N}$. 
The first few polynomials $A_{0}, A_{1}, \ldots$ are

$$
\begin{aligned}
A_{0}= & 1 \\
A_{1}= & \frac{11}{24}-\frac{x}{12} \\
A_{2}= & \frac{265}{1152}-\frac{47 x}{288}+\frac{x^{2}}{288} \\
A_{3}= & \frac{48703}{414720}-\frac{3649 x}{13824}+\frac{107 x^{2}}{6912}+\frac{139 x^{3}}{51840} \\
A_{4}= & \frac{2333717}{39813120}-\frac{2019163 x}{4976640}+\frac{16489 x^{2}}{331776}+\frac{26549 x^{3}}{1244160}-\frac{571 x^{4}}{2488320} \\
A_{5}= & \frac{38180761}{1337720832}-\frac{293093189 x}{477757440}+\frac{16859263 x^{2}}{119439360}+ \\
& +\frac{6752203 x^{3}}{59719680}-\frac{170729 x^{4}}{59719680}-\frac{163879 x^{5}}{209018880}
\end{aligned}
$$

The coefficients $B_{k}$ of the formal power series $\sum_{k=1}^{\infty} B_{k}(x) t^{k}=\log \left(\sum_{k=0}^{\infty} A_{k}(x) t^{k}\right)$ seem to be simpler and start as

$$
\begin{aligned}
& B_{1}=\frac{11}{24}-\frac{x}{12} \\
& B_{2}=\frac{1}{8}-\frac{x}{8} \\
& B_{3}=\frac{127}{2880}-\frac{3 x}{16}+\frac{x^{2}}{288}+\frac{x^{3}}{360} \\
& B_{4}=\frac{1}{64}-\frac{9 x}{32}+\frac{11 x^{2}}{576}+\frac{x^{3}}{48} \\
& B_{5}=\frac{221}{40320}-\frac{27 x}{64}+\frac{41 x^{2}}{576}+\frac{1381 x^{3}}{12960}-\frac{x^{4}}{1440}-\frac{x^{5}}{1260}
\end{aligned}
$$

Remark 27. The constant $1-\log 2=.30685281944 \ldots$ appearing in Conjecture 26 seems also to be related to the index $m_{n}$ such that $t_{m_{n}, 0}(n)=\max _{i}\left(t_{i, 0}(n)\right)$ with $m_{n}$ given asymptotically by $\frac{n}{2(1-\log 2)}$. Moreover, we have seemingly $\lim _{n \rightarrow \infty} \frac{t_{m_{n}, 0}(n) \sqrt{n}}{s(n)} \sim .87$ (and the numbers $t_{i, 0}(n)$, suitably rescaled, should satisfy a central limit Theorem).

\section{Modular properties of the sequence $s(1), s(2), \ldots$}

Proposition 28. The series $U\left(x, \sigma_{1}, \sigma_{2}, \ldots\right) \in \mathbb{F}_{p}[[x]]$ is rational if $\sigma_{1}, \sigma_{2}, \ldots$ is an ultimately periodic sequence of elements in $\mathbb{F}_{p}$.

Proof. Up to addition of a polynomial to $U=U\left(x, \sigma_{1}, \sigma_{2}, \ldots\right)$ we can suppose that $\sigma_{2}, \sigma_{3}, \sigma_{4}, \ldots$ is periodic with period $k$. We set $\tilde{\sigma}_{i}=\sigma_{i}$ for $i \geqslant 2$ and extend $\tilde{\sigma}_{2}, \tilde{\sigma}_{3}, \ldots$, to 
a $k$-periodic sequence indexed by $\mathbb{Z}$. We suppose first $\sigma_{1} \neq 0$ in $\mathbb{F}_{p}$. Using the identity $\left(-\sigma_{1}\right)^{p-1}=1$ and periodicity of the sequence $\left(\tilde{\sigma}_{i}\right)_{i \in \mathbb{Z}}$, we have

$$
\begin{aligned}
U & =1-\sum_{n=1}^{\infty} x^{n} \sum_{i=0}^{p k-1} \sum_{\alpha \in \mathbb{Z}} \tilde{\sigma}_{i+\alpha k p} \sum_{j=0}^{p-2} \sum_{\beta \in \mathbb{Z}} t_{i+\alpha k p, j+\beta(p-1)}(n)\left(-\sigma_{1}\right)^{j+\beta(p-1)} \\
& =1-\sum_{n=1}^{\infty} x^{n} \sum_{i=0}^{p k-1} \tilde{\sigma}_{i} \sum_{j=0}^{p-2}\left(-\sigma_{1}\right)^{j}\left(\sum_{\alpha \in \mathbb{Z}} \sum_{\beta \in \mathbb{Z}} t_{i+\alpha k p, j+\beta(p-1)}(n)\right)
\end{aligned}
$$

Since the recurrence relations 2 define the elements $t_{i, j}(2), t_{i, j}(3), \ldots$ correctly for arbitrary indices $i, j \in \mathbb{Z}$ we have

$$
\begin{aligned}
& \sum_{\alpha \in \mathbb{Z}} \sum_{\beta \in \mathbb{Z}} t_{i+\alpha k p, j+\beta(p-1)}(n) \\
= & (i-2) \sum_{\alpha \in \mathbb{Z}} \sum_{\beta \in \mathbb{Z}} t_{i-1+\alpha k p, j+\beta(p-1)}(n-1) \\
& +\sum_{\alpha \in \mathbb{Z}} \sum_{\beta \in \mathbb{Z}} t_{i-1+\alpha k p, j-1+\beta(p-1)}(n-1) \\
& +(i-3) \sum_{\alpha \in \mathbb{Z}} \sum_{\beta \in \mathbb{Z}} t_{i-2+\alpha k p, j+\beta(p-1)}(n-1)
\end{aligned}
$$

for $n \geqslant 2$. Setting

$$
\tilde{t}_{i, j}(n) \equiv \sum_{\alpha \in \mathbb{Z}} \sum_{\beta \in \mathbb{Z}} t_{i+\alpha k p, j+\beta(p-1)}(n-1)
$$

for $0 \leqslant i<k p$ and $0 \leqslant j<p-1$, the elements $\tilde{t}_{i, j}(n)$ of $\mathbb{F}_{p}$ satisfy the recursion relation 2 with indices considered modulo $k p$ for $i$ and modulo $p-1$ for $j$. Since the $k p(p-1)$ elements $\tilde{t}_{i, j}(n)$ of the finite field $\mathbb{F}_{p}$ depend affinely on the $k p(p-1)$ elements $\tilde{t}_{i, j}(n-1)$ for $n \geqslant 2$, finiteness of the set $(i, j)$ of indices implies the existence of an integer $l$ such that $\tilde{t}_{i, j}(n+l)=t_{i, j}(n)$ for all sufficiently large $n$ and for all possible indices $i$ and $j$. This implies easily that the coefficients of $U$ are ultimately periodic and ends the proof for $\sigma_{1} \neq 0$.

The case $\sigma_{1}=0$ involves only the integers $t_{i, 0}(n)$ and their analogues $\tilde{t}_{i, 0}(n)$ with indices in the finite set $\{0, \ldots, p k-1\}$. Details are similar to the previous case and left to the reader.

The first non-trivial case of Proposition 28 is perhaps given by the generating series $U(x, 0,-1,-1,-1, \ldots)$ with coefficients of $U(x, 0,-1,-1, \ldots)-1$ given by the sequence

$$
s(n)=\sum_{i=n+1}^{2 n} t_{i, 0}(n)
$$

obtained by summing all coefficients in the first column of the triangular arrays $T(1), T(2), \ldots$. 
Conjecture 29. There exists a sequence

$$
\begin{aligned}
& \alpha_{0}=-1, \alpha_{1}=2, \alpha_{2}=0, \alpha_{3}=\frac{1}{3}, \alpha_{4}=\frac{5}{18}, \alpha_{5}=\frac{149}{540}, \alpha_{6}=\frac{553}{2025}, \\
& \alpha_{7}=\frac{1849741}{6804000}, \alpha_{8}=\frac{775167119}{2857680000}, \alpha_{9}=\frac{325214957371}{1200225600000}, \ldots
\end{aligned}
$$

of rational numbers such that

$$
\left(1+x^{p-1}\right) \sum_{n=1}^{\infty} s(n) x^{n} \equiv x+\sum_{n=0}^{p-2} \alpha_{n} x^{p-n} \quad(\bmod p)
$$

for every prime number $p$.

Conjecture 30. The rational sequence $\alpha_{0}, \alpha_{1}, \ldots$ has an asymptotic expansion given by

$$
\alpha_{n} \sim \sum_{k=1}^{\infty} \frac{k^{k-n}}{k !}\left(\frac{2}{e^{2}}\right)^{k}
$$

and converges with limit given by $2 e^{-2}=.27067056647322538378799 \ldots$.

The error term

$$
\epsilon_{n}=\alpha_{n}-\sum_{k=1}^{\infty} \frac{k^{k-n}}{k !}\left(\frac{2}{e^{2}}\right)^{k}
$$

is given by

$$
\epsilon_{n}=\frac{(-1)^{n+1}}{(1-\log 2) s(n+1)}\left(\sum_{k=0}^{m} \frac{\gamma_{2 k}(1-\log 2)}{n^{2 k}}+o\left(n^{-2 m-1}\right)\right)
$$

where $\gamma_{2 k}(x) \in \mathbb{Q}[x]$ is a polynomial of degree at most $2 k$. The first few polynomials are given by

$$
\begin{aligned}
& \gamma_{0}=1 \\
& \gamma_{2}=-\frac{x}{12} \\
& \gamma_{4}=-\frac{x}{48}+\frac{x^{2}}{48}+\frac{x^{3}}{40} \\
& \gamma_{6}=-\frac{x}{192}+\frac{5 x^{2}}{96}+\frac{193 x^{3}}{864}-\frac{x^{4}}{72}-\frac{5 x^{5}}{252}
\end{aligned}
$$

\section{Integer sequences obtained as weighted sums of the numbers $t_{i, j}(n)$}

The sequence $q_{i}(x, y)$ defined by

$$
q_{i}(x, y)=\sum_{n, j \geqslant 0} t_{i, j}(n) x^{n} y^{j}=\sum_{n=\lceil i / 2\rceil}^{i-1} x^{n} \sum_{j=0}^{n-1} t_{i, j}(n) y^{j}
$$


is given by $q_{1}(x, y)=0, q_{2}(x, y)=x$ and by the recursion relation

$$
q_{i}(x, y)=x\left((i-2+y) q_{i-1}(x, y)+(i-3) q_{i-2}(x, y)\right)
$$

for $i \geqslant 3$. The following table lists the first few non-zero coefficients $q_{2}(x, y), q_{3}(x, y), \ldots$ (up to normalizations) and the seemingly corresponding sequences of [4] (which have often interesting combinatorial interpretations) for a few specializations:

\begin{tabular}{|l|c|c|}
\hline$q_{i}(-3,-2 / 3)(-1)^{i+1} / 3$ & $1,1,1,1,1,1, \ldots$ & $A 12$ \\
\hline$q_{i}(-3,1 / 3)(-1)^{i+1} / 3$ & $1,4,25,226,2713,40696, \ldots$ & $A 10845$ \\
\hline$q_{i}(-2,-3 / 2)(-1)^{i+1} / 2$ & $1,-1,-3,-5,-7,-9,-11, \ldots$ & \\
\hline$q_{i}(-2,-1 / 2)(-1)^{i+1} / 2$ & $1,1,1,1,1,1, \ldots$ & $A 12$ \\
\hline$q_{i}(-2,1 / 2)(-1)^{i+1} / 2$ & $1,3,13,79,633,6331, \ldots$ & $A 10844$ \\
\hline$q_{i}(-1,-1)(-1)^{i+1}$ & $1,0,-1,-2,-3,-4,-5, \ldots$ & \\
\hline$q_{i}(-1,0)(-1)^{i+1}$ & $1,1,1,1,1,1, \ldots$ & $A 12$ \\
\hline$q_{i}(-1,1)(-1)^{i+1}$ & $1,2,5,16,65,326,1957, \ldots$ & $A 522$ \\
\hline$q_{i}(-1,2)(-1)^{i+1}$ & $1,3,11,49,261,1631, \ldots$ & $A 1339$ \\
\hline$q_{i}(-1,3)(-1)^{i+1}$ & $1,4,19,106,685,5056, \ldots$ & $A 82030$ \\
\hline$q_{i}(1,-2)(-1)^{i}$ & $1,1,1,1,1,1, \ldots$ & $A 12$ \\
\hline$q_{i}(1,-1)$ & $1,0,1,2,9,44,265,1854, \ldots$ & $A 166$ \\
\hline$q_{i}(1,0)$ & $1,1,3,11,53,309,2119, \ldots$ & $A 255$ \\
\hline$q_{i}(1,1)$ & $1,2,7,32,181,1214, \ldots$ & $A 153$ \\
\hline$q_{i}(1,2)$ & $1,3,13,71,465,3539, \ldots$ & $A 261$ \\
\hline$q_{i}(1,3)$ & $1,4,21,134,1001,8544, \ldots$ & $A 1909$ \\
\hline$q_{i}(2,-7 / 2)(-1)^{i} / 2$ & $1,3,37,65,101,145,197, \ldots$ & $A 53755$ \\
\hline$q_{i}(2,-5 / 2)(-1)^{i} / 2$ & $1,1,1,1,1,1, \ldots$ & $A 5408$ \\
\hline$q_{i}(2,-3 / 2)(-1)^{i} / 2$ & $1,0,2,8,60,544,6040, \ldots$ & $A 53871$ \\
\hline$q_{i}(2,-1) / 2$ & $1,1,5,29,233,2329, \ldots$ & $A 354$ \\
\hline$q_{i}(2,-1 / 2) / 2$ & $1,1,1,1,1,1, \ldots$ & $A 12$ \\
\hline$q_{i}(3,-4 / 3)(-1)^{i} / 3$ & $1,0,3,18,189,2484, \ldots$ & $A 33030$ \\
\hline$q_{i}(3,-1) / 3$ & & \\
\hline
\end{tabular}

The sequences A255, A153, A261 and A1909 corresponding to $q_{i}(1,0), q_{i}(1,1), q_{i}(1,2)$ and $q_{i}(1,3)$ can seemingly also be obtained by considering the weighted sums

$$
s_{i}=\sum_{j, n \geqslant 0}\left(\begin{array}{c}
n-j+k-1 \\
k
\end{array}\right) t_{i, j}(n)(-1)^{j}
$$

for $k=1,2,3$ and 4 .

The above table contains a few instances of the identities

$$
1=q_{i}\left(\kappa,-\frac{\kappa+1}{\kappa}\right) \frac{(-1)^{i}}{\kappa}
$$

and

$$
1+(i-2) \kappa=q_{i}\left(\kappa,-\frac{2 \kappa+1}{\kappa}\right) \frac{(-1)^{i}}{\kappa}
$$


which hold for $\kappa \neq 0$ and for $i=2,3,4, \ldots$ and which can easily be proven by induction. (These two examples generalize probably to $q_{i}\left(\kappa,-\frac{\lambda \kappa+1}{\kappa}\right) \frac{(-1)^{i}}{\kappa}=P_{\lambda}(i, \kappa), \lambda=$ $1,2,3, \ldots, \kappa \neq 0, i=2,3,4, \ldots$ for $P_{\lambda}$ a suitable polynomial function of $\kappa$ and $i$.)

Another identity is given by the family of weighted examples

$$
1=(-1)^{i} k !(i-1+k) \sum_{j, n \geqslant 0} t_{i, j}(n) \frac{(-1)^{j}}{(n+k-j) !}
$$

for all $k \in \mathbb{N}$ and for all $i \geqslant 2$.

A few other interesting weighted examples (there are probably many more) are given by the following formulae

$$
\begin{aligned}
a_{i} & =\sum_{j, n \geqslant 0}(n-j) t_{i, j}(n)(-2)^{j}, \\
b_{i} & =\frac{1}{4} \sum_{j, n \geqslant 0}(n-1-j) t_{i, j}(n) 2^{n}\left(\frac{-3}{2}\right)^{j}, \\
c_{i} & =\sum_{j, n \geqslant 0} t_{i, j}(n)(n-1-j) !(-1)^{j}, \\
d_{i} & =(-1)^{i+1} \sum_{j, n \geqslant 0} t_{i, j}(n)(n-1-j) !(-1)^{n}, \\
e_{i} & =\sum_{j, n \geqslant 0} t_{i, j}(n) \frac{(-1)^{j}}{(n-1-j) !}, \\
f_{i} & =(i-1) ! \sum_{n \geqslant 1} \frac{t_{i, 0}(n)}{(n-1) !} .
\end{aligned}
$$

Their initial coefficients (with leading zeros omitted) and the seemingly corresponding sequences of [4] are as follows:

\begin{tabular}{|c|l|c|}
\hline$a_{i}$ & $1,0,0,1,1,8,36,229,1625, \ldots$ & $A 757$ \\
\hline$b_{i}$ & $1,0,5,24,209,2120, \ldots$ & $A 120765$ \\
\hline$c_{i}$ & $1,0,3,26,453,11844, \ldots$ & $A 89041$ \\
\hline$d_{i}$ & $1,2,7,52,749,17686, \ldots$ & $A 46662$ \\
\hline$e_{i}$ & $1,0,0,0,0,0,0, \ldots$ & $A 7$ \\
\hline$f_{i}$ & $1,2,12,84,820,9540, \ldots$ & $A 179495$ \\
\hline
\end{tabular}

Most of the proofs are probably easy: The corresponding sections of [4] give information concerning generating functions which can be applied to differential equations analogous to those of Section 8.2.

\section{Coverings}

Coverings and packings are dual notions. We discuss here a few aspects of the theory of coverings in relation with packings by generic families. 
A (left-) covering with parts $\mathcal{S}_{1}, \ldots, \mathcal{S}_{n}$ of a group $G$ is a vector $\left(g_{1}, \ldots, g_{n}\right)$ such that $G=\cup_{j=1}^{n} g_{j} \mathcal{S}_{j}$.

A covering of a finite group $G$ with $N$ elements by non-empty subsets $\mathcal{S}_{1}, \ldots, \mathcal{S}_{n}$ exists of course always if $n \geqslant N$.

We are interested in large collections of subsets $\mathcal{S}_{1}, \ldots, \mathcal{S}_{n}$ in a finite group $G$ of order $N$ such that the sets $\mathcal{S}_{1}, \ldots, \mathcal{S}_{n}$ (or more precisely, suitable translates) cover $G$ and the number of all coverings depends only on the cardinalities of $\mathcal{S}_{1}, \ldots, \mathcal{S}_{n}$ (and of $N$ ) for every family $\mathcal{S}_{1}, \ldots, \mathcal{S}_{n}$ in the collection.

Three such collections can be described as follows:

Start with a family $\mathcal{S}_{1}, \ldots, S_{n}$ which is generic for packings and add $N-\sum_{j=1}^{n} \sharp\left(\mathcal{S}_{j}\right)$ singletons. Coverings of $G$ by such families are "tight" and essentially in one-to-one correspondence (except for a factor $\left(N-\sum_{j=1}^{n} \sharp\left(\mathcal{S}_{j}\right)\right)$ ! accounting for all permutations of the added singletons) with packings by $\mathcal{S}_{1}, \ldots, \mathcal{S}_{n}$.

The second family is obtained by adding $N+n-1-\sum_{j=1}^{n} \sharp\left(\mathcal{S}_{j}\right)$ singletons to a family $\mathcal{S}_{1}, \ldots, S_{n}$ which is generic for packings. The fact that the number of associated coverings depends only on all involved cardinalities is similar to the proof of Proposition 5 given in Section 4. The proof needs probably computations with the full Möbius function. I do not know if there is an efficient way for computing the number of associated coverings or if there is a nice formula similar to the one assciated to enumerations of packings.

There is a further variation on this theme: Given an arbitrary natural integer $a$ one can consider adding $N+a-\sum_{j=1}^{n} \sharp\left(\mathcal{S}_{j}\right)$ singletons to a family $\mathcal{S}_{1}, \ldots, S_{n}$ which is generic for packings. For every natural integer $a$, such a family has the property that the number of associated coverings depends only on all involved cardinalities. The choices $a=0$ corresponding to the first family and $a=n-1$ corresponding to the second family are however natural in this context. Indeed, since the intersection graph of the sets $g_{1} \mathcal{S}_{1}, \ldots, g_{n} \mathcal{S}_{n}$ is a hyperforest, the union $\cup_{j=1}^{n} g_{j} \mathcal{S}_{j}$ contains at least $\sum_{j=1}^{n} \sharp\left(\mathcal{S}_{j}\right)-(n-1)$ elements. This leaves at most $N+n-1-\sum_{j=1}^{n} \sharp\left(\mathcal{S}_{j}\right)$ missing elements which can be covered using the additional singletons.

A third rather trivial family is given by considering complements $G \backslash \mathcal{S}_{1}, \ldots, G \backslash \mathcal{S}_{n}$ where $\mathcal{S}_{1}, \ldots, \mathcal{S}_{n}$ is a generic family for packings in $G$ having at least two parts. The number of coverings of such a family is easy to compute and given by $N^{n}-N \prod_{j=1}^{n} \sharp\left(\mathcal{S}_{j}\right)$.

It would perhaps be interesting to have other (and hopefully more exotic) families of examples.

Acknowledgements. I thank Pierre de la Harpe for helpful comments and two anonymous referees for their careful work and useful remarks.

\section{References}

[1] I. M. Gessel, L. H. Kalikow. Hypergraphs and a functional equation of Bouwkamp and de Bruijn. J. Combinatorial Theory Ser. A, 12(2):275-289, 2005. 
[2] K. Husimi. Note on Mayer's theory of cluster integrals. Journal of Chemical Physics, 18:682-684, 1950.

[3] J. McCammond, J. Meier. The hypertree poset and the $l^{2}$-Betti numbers of the motion group of the trivial link. Math. Ann., 328(4):633-652, 2004.

[4] N. J. A. Sloane. The On-Line Encyclopedia of Integer Sequences. Published electronically at http://oeis.org.

[5] R. P. Stanley. Enumerative Combinatorics, volume I. Cambridge University Press, 1997. 